\title{
A GENERALIZED EXPANSION METHOD FOR NON-LINEAR WAVE EQUATIONS
}

\author{
Qun Lin*, Yong Hong Wu and Ryan Loxton \\ Department of Mathematics and Statistics, \\ Curtin University of Technology, Perth, Australia.
}

\begin{abstract}
A generalized Jacobian/exponential expansion method for finding the exact traveling wave solutions of a non-linear partial differential equation is discussed. We use this method to construct many new, previously undiscovered exact solutions for the Boussinesq and modified $\mathrm{KdV}$ equations. We also apply it to the shallow long wave approximate equations. New solutions are deduced for this system of partial differential equations.
\end{abstract}

Keywords: Boussinesq equation; Modified KdV equation; Shallow long wave approximate equations; Traveling wave solutions; Jacobian elliptic solutions.

\footnotetext{
*Corresponding Author: Q.Lin@curtin.edu.au
} 


\section{Introduction}

Solutions of partial differential equations have attracted significant interest in the literature. Exact traveling wave solutions, in particular, are useful both in practice and for verifying the accuracy and stability of popular numerical schemes such as the finite difference and finite element methods. By employing a computer algebra software such as Maple or Mathematica, the large amounts of tedious working required to verify candidate traveling wave solutions can be avoided. The capability and power of these softwares has increased dramatically over the past decade. Hence, a direct search for exact solutions is now much more viable.

Several effective direct search methods have been proposed in the literature. These include the tanh method [15,16], Exp-function method [6,20], Jacobian elliptic function method [12,18], Weierstrass's elliptic function method [17], reduction of order methods [9,10], and cosh/sinh ansatz I-III method [19]. In this paper, we extend the generalized expansion method developed in references [2,3]. More specifically, we develop some new Jacobian elliptic and exponential solution classes for the same auxiliary ordinary differential equation (ODE) considered in these papers. The solutions of the ODE are then used to construct candidate traveling wave solutions. Our new results ensure that, when applied to the classical Boussinesq and modified KdV equations, this generalized expansion method not only recovers all of the solutions reported in $[6,12,18,19,22]$, but also discovers many new ones. Furthermore, this approach is flexible as well as powerful - it is easily adapted in Section 6 to handle the system of shallow long wave approximate equations.

\section{Preliminary results}

The Jacobian elliptic functions are discussed thoroughly in $[1,5]$. Since these special functions play an important role in the sequel, we will briefly introduce them here. We will also discuss some preliminary results that form the basis for our work in Sections 
3-6. Note that we will follow the usual convention and let i denote the complex number satisfying $\mathrm{i}^{2}=-1$. Moreover, for the remainder of this paper, $m \in(0,1)$ is arbitrary.

To begin, consider the integral

$$
\zeta=\underbrace{\rho}_{0} \frac{\lambda d \eta}{1-m^{2} \sin ^{2}(\eta)}
$$

Here, the constant $m$ is referred to as the modulus and the upper limit $\rho$ is called the amplitude of $\zeta$, which we denote as

$$
\rho=\operatorname{am}(\zeta)
$$

On this basis, the first three Jacobian elliptic functions are defined as

$$
\begin{aligned}
& \operatorname{sn}(\zeta):=\sin [\operatorname{am}(\zeta)]=\sin (\rho), \\
& \operatorname{cn}(\zeta):=\cos [\operatorname{am}(\zeta)]=\cos (\rho),
\end{aligned}
$$

and

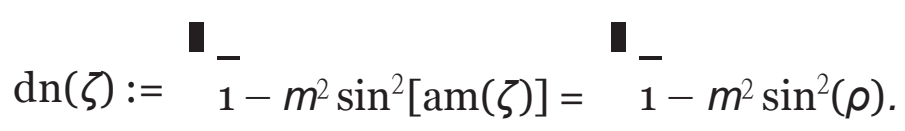

As $m \rightarrow 1$, we have

$$
\operatorname{sn}(\zeta) \rightarrow \tanh (\zeta), \quad \operatorname{cn}(\zeta) \rightarrow \operatorname{sech}(\zeta), \quad \operatorname{dn}(\zeta) \rightarrow \operatorname{sech}(\zeta)
$$

Similarly, as $m \rightarrow 0$,

$$
\operatorname{sn}(\zeta) \rightarrow \sin (\zeta), \quad \operatorname{cn}(\zeta) \rightarrow \cos (\zeta), \quad \operatorname{dn}(\zeta) \rightarrow 1 .
$$

Nine additional Jacobian elliptic functions can be defined in terms of these first three 
- see references $[1,5]$ for details.

In $[2,3]$, the following auxiliary ODE was introduced:

$$
\left[F^{1}(\xi)\right]^{2}=q_{0}+q_{1} F(\xi)+q_{2}[F(\xi)]^{2}+q_{3}[F(\xi)]^{3}+q_{4}[F(\xi)]^{4}
$$

where $q_{k}, k=0, \ldots, 4$, are given coefficients. Various solutions of ODE (2.1) were constructed using the Jacobian elliptic functions, and these results were exploited in the design of a systematic procedure for generating solutions of non-linear partial differential equations. We will follow a similar approach in this paper. In our work, ODE (2.1) will be considered assuming $q_{4} /=0$. We will need to determine more general solution classes of ODE $(2.1)$ than those reported in $[2,3]$. This is the motivation behind the preliminary results that follow.

Recall that $m$ is an arbitrary real number satisfying $0<m<1$. With this in mind, for any (possibly complex) number $\gamma$, define the constants $p_{j, k}(\gamma), j=1, \ldots, 12$, $k=0, \ldots, 4$, according to the following table.

\begin{tabular}{|c|c|c|c|c|c|}
\hline$j$ & $p_{\mathrm{j}, \mathrm{O}}(V)$ & $p_{\mathrm{j}, 1}(V)$ & $p_{\mathrm{j}, 2}(V)$ & $p_{\mathrm{j}, 3}(V)$ & $p_{\mathrm{j}, 4}(\gamma)$ \\
\hline 1 & $m^{2}-1$ & $4 \gamma\left(1-m^{2}\right)$ & $2-6 \gamma^{2}+6 \gamma^{2} m^{2}-m^{2}$ & $2 \gamma\left(2 \gamma^{2}-2+m^{2}-2 \gamma^{2} m^{2}\right)$ & $v^{4} m^{2}+2 \gamma^{2}-\gamma^{4}-1-v^{2} m^{2}$ \\
\hline 2 & 1 & $-4 y$ & $6 y^{2}-1-m^{2}$ & $2 \gamma\left(1+m^{2}-2 \gamma^{2}\right)$ & $v^{4}+m^{2}-v^{2}-v^{2} m^{2}$ \\
\hline 3 & $1-m^{2}$ & $4 \gamma\left(m^{2}-1\right)$ & $2 m^{2}-6 y^{2} m^{2}+6 \gamma^{2}-1$ & $2 \gamma\left(2 \gamma^{2} m^{2}-2 \gamma^{2}+1-2 m^{2}\right)$ & $2 \gamma^{2} m^{2}+\gamma^{4}-m^{2}-\gamma^{4} m^{2}-\gamma^{2}$ \\
\hline 4 & $-\frac{1}{4}$ & $Y$ & $\frac{-3 \gamma^{2}+1-2 m^{2}}{2}$ & $\gamma\left(2 m^{2}+V^{2}-1\right)$ & $\frac{-\gamma^{4}-1-4 \gamma^{2} m^{2}+2 \gamma^{2}}{4}$ \\
\hline 5 & $-\frac{1}{4}$ & Y & $\frac{1-3 \gamma^{2}+m^{2}}{2}$ & $\gamma\left(V^{2}-1-m^{2}\right)$ & $\frac{2 v^{2}+2 m^{2}-v^{4}-1+2 v^{2} m^{2}-m^{4}}{4} 2 v m$ \\
\hline 6 & $-\frac{m^{2}}{4}$ & $\gamma m^{2}$ & $\frac{m^{2}-3 \gamma^{2} m^{2}-2}{2}$ & $V\left(V^{2} m^{2}-m^{2}+2\right)$ & $\begin{array}{lllll}2 & 2 & 4 & 2 & 2 \\
\end{array}$ \\
\hline 7 & 0 & $m^{2}-1$ & $3 y+2-3 y m^{2}-m^{2}$ & $3 y^{2} m^{2}+2 y m^{2}-3 y^{2}-4 y-1$ & $\gamma(V+1)\left(V+1-\gamma m^{2}\right)$ \\
\hline 8 & 0 & $-2 \sqrt{1-m^{2}}$ & $6-\overline{1-m^{2} V-4 m^{2}+5}$ & $\left(8 m^{2}-10\right) y-\left(6 V^{2}+4\right) \overline{1-m^{2}}$ & $\left(4 \gamma+2 V^{3}\right)^{P} \overline{1-m^{2}}+1+\left(5-4 m^{2}\right) \gamma^{2}$ \\
\hline 9 & $\begin{array}{l}1 \\
4\end{array}$ & $\underline{0}+$ & $-m^{2}+2$ & 0 & $\begin{array}{l}1 \\
4\end{array}$ \\
\hline 10 & $\frac{m^{2}}{4\left(1-m^{2}\right)}$ & $\begin{array}{c} \\
m^{2}-1\end{array}$ & $\frac{2 m^{4}-3 m^{2}+4}{2\left(1-m^{2}\right)}$ & $\begin{array}{l}2 \\
m^{2}-1\end{array}$ & $\frac{m^{2}}{4\left(1-m^{2}\right)}$ \\
\hline 11 & $\begin{array}{l}1-m^{2} \\
4\end{array}$ & 0 & $\begin{array}{c}\overline{1+m^{2}} \\
2\end{array}$ & 0 & $\begin{array}{l}1-m^{2} \\
4\end{array}$ \\
\hline 12 & $\begin{array}{l}\frac{m m_{2}\left(2-m_{2}\right)}{2} \\
4\left(1-m^{2}\right)\end{array}$ & $\begin{array}{c}4 \\
m^{2}-1\end{array}$ & $\frac{m_{1}-4}{4}$ & $\begin{array}{l}m_{4}+m_{1} \\
m^{2}-1\end{array}$ & $\begin{array}{l}\frac{m m_{2}\left(2-m m_{2}\right)}{2} \\
4\left(1-m^{2}\right)\end{array}$ \\
\hline
\end{tabular}

Table 1. Definition of the constants $\boldsymbol{p}_{\mathrm{j}, \mathrm{k}}(\boldsymbol{\gamma}), \boldsymbol{j}=1, \ldots, 12, \boldsymbol{k}=0, \ldots, 4$.

Furthermore, let the functions $\phi_{j, k}(\cdot, \gamma), j=1, \ldots, 12, k=1, \ldots, 4$, be defined as follows: 


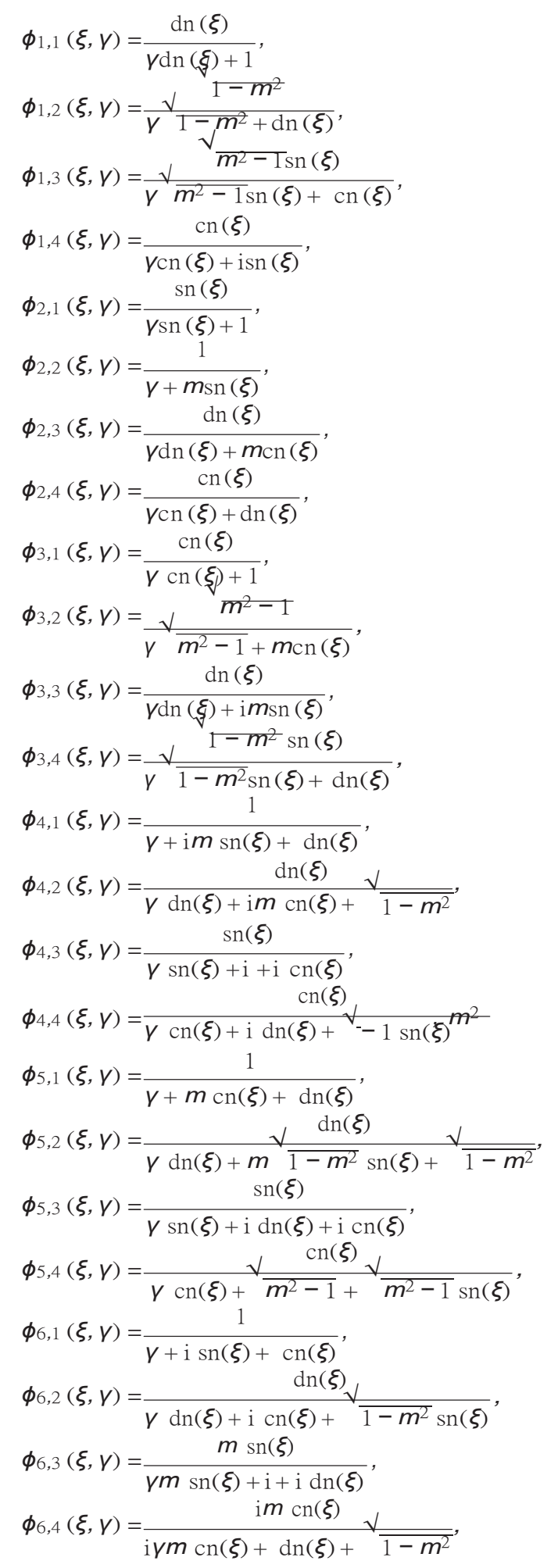




$$
\begin{aligned}
& \phi_{7,1}(\xi, v)=\frac{\sqrt{V}}{V 1-m^{2}+\frac{\sqrt{1-m^{2}}[1+\operatorname{sn}(\xi)]}{1-m^{2}(\gamma+1) \operatorname{sn}(\xi)+\operatorname{dn}(\xi)},} \\
& \phi_{7,2}(\xi, \gamma)=\frac{\operatorname{dn}(\xi)+\operatorname{cn}(\xi)}{V \operatorname{dn}(\xi)+(V+1) \operatorname{cn}(\xi)+1},
\end{aligned}
$$

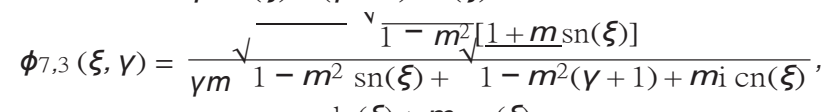

$$
\begin{aligned}
& \phi_{7,4}(\xi, \gamma)=\frac{\operatorname{dn}(\xi)+m \operatorname{cn}(\xi)}{m \gamma \operatorname{cn}(\xi)+(\bigvee+1) \operatorname{dn}(\xi)+\mathrm{i} m \operatorname{sn}(\xi)}, \\
& \phi_{8,1}(\xi, \gamma)=\frac{\sqrt[d n]{ }(\xi)+\overline{1-m^{2}} \operatorname{sn}(\xi)}{\left(1+\sqrt{\left.1-m^{2} \gamma\right) \operatorname{sn}(\xi)+\gamma \operatorname{dn}(\xi)}\right.},
\end{aligned}
$$

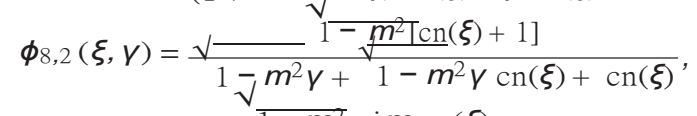

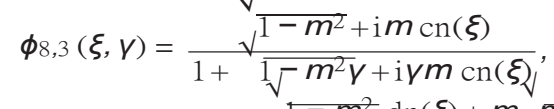

$$
\begin{aligned}
& \phi_{8,4}(\xi, \gamma)=\frac{1-m^{2} \operatorname{dn}(\xi)+m m^{2}-1 \operatorname{sn}(\xi)}{\operatorname{dn}(\xi)+V \sqrt{1-m^{2} \operatorname{dn}(\xi)+m} \overline{m^{2}-1 V} \operatorname{sn}(\xi)}, \\
& \phi_{9,1}(\xi, \gamma)=\frac{\sqrt{ } \frac{\mathrm{sn}(\xi)}{m 2-m^{2}+}+\frac{\sqrt{-m^{2}} \mathrm{dn}(\xi)}{-m^{4}+m^{2}+1} \mathrm{cn}(\xi)}{,}
\end{aligned}
$$

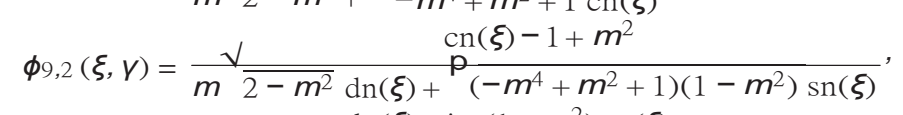

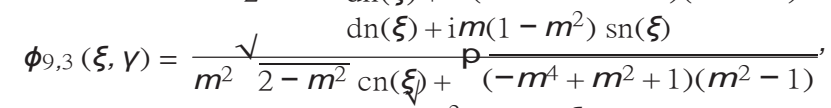

$$
\begin{aligned}
& \phi_{9,4}(\xi, \gamma)=\frac{\sqrt{ } \frac{1+m}{m^{2}-1} \bar{v}^{2}(\xi)}{m^{2} 2-m^{2} \$ n(\xi)+\overline{m^{4}-m^{2}-1} \operatorname{dn}(\xi)}, \\
& \phi_{10,1}(\xi, \gamma)=\frac{\mathrm{cn}(\xi)+\sqrt{v} \frac{1-m^{2}}{\mathrm{dn}(\xi)}}{m^{2}-1+\sqrt{m^{4}-m^{2}+1} \mathrm{cn}(\xi)},
\end{aligned}
$$

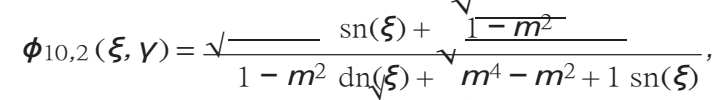

$$
\begin{aligned}
& \phi_{10,3}(\xi, v)=\frac{\sqrt{ } \frac{1+m}{m m^{2}-1 \mathrm{cn}(\xi)+\sqrt{ } \bar{m}^{2}} \operatorname{sn}(\xi)}{m^{4}-m^{2}+1}, \\
& \phi_{10,4}(\xi, \gamma)=\frac{\mathrm{dn}(\xi)+m \sqrt[1]{ }-m^{2} \operatorname{cn}(\xi)}{\mathrm{i}\left(m^{3}-m\right) \operatorname{sn}(\xi)+\overline{m^{4}-m^{2}+1} \operatorname{dn}(\xi)}, \\
& \phi_{11,1}(\xi, \gamma)=\frac{\operatorname{cn}(\xi)+\sqrt{1-m^{2}} \operatorname{dn}(\xi)}{m+\sqrt{m^{4}-m^{2}+1} \operatorname{sn}(\xi)}, \\
& \phi_{11,2}(\xi, \gamma)=\frac{1-m^{2}}{m \operatorname{dn}(\xi)+\sqrt{m^{4}-m^{2}+1 \operatorname{cn}(\xi)}}, \\
& \phi_{11,3}(\xi, \gamma)=\frac{i\left[\operatorname{dn}(\xi)+m \sqrt{ } \sqrt{-m^{2}} \mathrm{cn}(\xi)\right]}{m^{2} \operatorname{sn}(\xi)+\sqrt[m^{4}-m^{2}+1]{\sqrt{m^{2}-1}}}, \\
& \phi_{11,4}(\xi, \gamma)=\frac{\overline{m^{2}-1}\left[1+m^{1-m^{2}} \operatorname{sn}(\xi)\right]}{m^{2} \operatorname{cn}(\xi)+\overline{m^{4}-m^{2}+1} \operatorname{dn}(\xi)},
\end{aligned}
$$

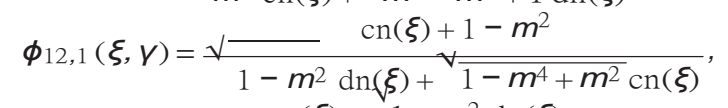

$$
\begin{aligned}
& \phi_{12,2}(\xi, y)=\frac{\sqrt{ } \frac{\operatorname{sn}(\xi)}{1-m^{2}+\sqrt{1-m^{2}} \operatorname{dn}(\xi)}}{1-\sqrt{1-m^{4}+m^{2}} \operatorname{sn}(\xi)},
\end{aligned}
$$

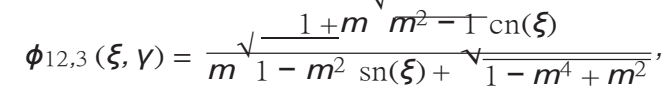

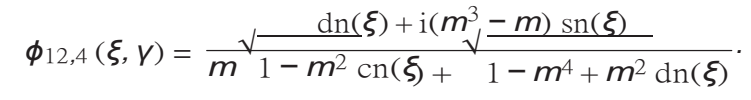


Through lengthy calculation, we can readily verify the following result. Note that Maple can be used to help us for the calculation.

Theorem 1. Let $\gamma$ be arbitrary. Then, for each $j=1, \ldots, 12$, ODE (2.1) with coefficients $q_{k}=p_{j, k}(\gamma), k=0, \ldots, 4$, has solutions $\phi_{j, k}(\cdot, \gamma), k=1, \ldots, 4$.

Remark 1. Theorem 1 can be generalized further. In fact, it remains valid even if $\operatorname{cn}(\xi), \operatorname{sn}(\xi)$ and $\operatorname{dn}(\xi)$ are replaced, respectively, by $\pm \operatorname{cn}(\xi), \pm \operatorname{sn}(\xi)$ and $\pm \operatorname{dn}(\xi)$ in the expressions for $\phi_{j, k}$ given above.

In some cases, the solutions of ODE (2.1) can be used to generate additional solutions. This observation is furnished precisely in Theorems 2 and 3 below. Again, Maple can be used to conveniently verify these results.

Theorem 2. Suppose that $\phi$ is a solution of ODE (2.1) with coefficients $q_{k}=\hat{q}_{k}$, $k=0, \ldots, 4$, where $\hat{q}_{1}=\hat{q}_{b}=0$, and $\hat{q}_{b}, \hat{q}_{2}$ and $\hat{q}_{A}$ are given constants such that $\hat{q}_{0} /=$ o. Then,

$$
\pm \begin{aligned}
& \overline{\hat{q}_{4}} \phi+\frac{1}{1} \\
& \hat{q} \phi
\end{aligned}
$$

is a solution of ODE (2.1) with coefficients

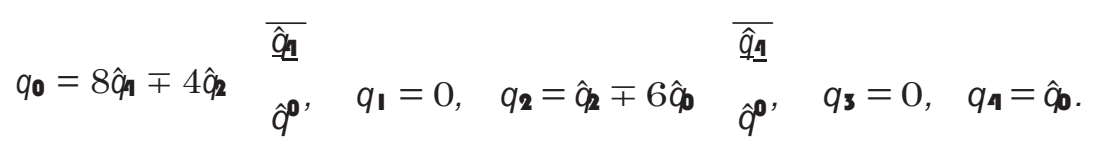

Theorem 3. Suppose that $\phi$ is a solution of ODE (2.1) with coefficients $q_{k}=\hat{q}_{k}$, $k=0, \ldots, 4$, where $\hat{q}_{k}, k=0, \ldots, 4$, are given constants such that $\hat{q}_{I} /=0$ and $\hat{q}_{4} \frac{\hat{q} \hat{q}}{\hat{q}^{3}}$. $=$ 
Then,

$$
\hat{\underline{q}}_{\hat{q}} \phi+\frac{1}{\phi}
$$

is a solution of ODE (2.1) with coefficients

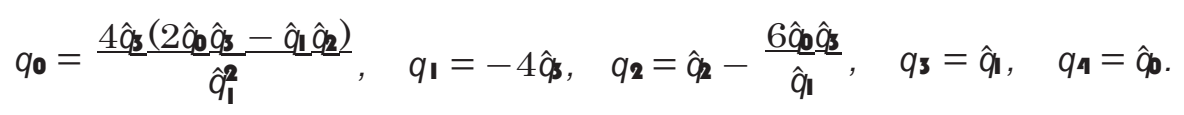

Remark 2. From Table 1 and Theorem 1, the reader will notice that, for any $\gamma$, Theorem 3 can be invoked with $\phi_{j, k}(\cdot, \gamma), j \in\{10,12\}, k=1, \ldots, 4$.

We would also like to consider non-Jacobian elliptic solutions of ODE (2.1). As such, to conclude this section, we present the following two results. Both can be proved easily via direct substitution.

Theorem 4. Let $a_{-1}, a_{0}, a_{1}$ and $b_{0}$ be given constants such that $a_{-1} /=0$ and $a_{0} /=a_{-1} b_{0}$. Then,

$$
\frac{a_{-1} \mathrm{e}^{-\xi}+a_{0}+a_{1} \mathrm{e}^{\xi}}{\mathrm{e}^{-\xi}+b_{0}+\frac{a_{1}}{a_{-1}} \mathrm{e}^{\xi}}
$$

is a solution of ODE (2.1) with coefficients

$$
\begin{aligned}
q_{0} & =-\frac{4 a-1 a_{1}-a_{0}^{2} a_{-1}^{2}}{\left(a-1 b_{0}-a_{0}\right)^{2}}, \\
q_{1} & =\frac{2 a-1-a_{0} a-1 b_{0}+8 a-1 a_{1}-a_{0}^{2}}{\left(a-1 b_{0}-a_{0}\right)^{2}}, \\
q_{2} & =\frac{a^{2} b_{0}^{2}+4 a-1 a_{0} b_{0}-24 a-1 a_{1}+a_{0}^{2}}{\left(a-1 b_{0}-a_{0}\right)^{2}}, \\
q_{3} & =\frac{2\left(8 a_{1}-a-1 b_{0}^{2}-a_{0} b_{0}\right)}{\left(a_{-1} b_{0}-a_{0}\right)^{2}}, \\
q_{4} & =\frac{a-1 b^{2}-4 a_{1}}{a-1\left(a-1 b_{0}-a_{0}\right)^{2}} .
\end{aligned}
$$

Theorem 5. Let $a_{-1}, a_{1}, b_{0}$ and $b_{1}$ be given constants such that $a_{1} /=b_{1} a_{-1}$ and 


$$
\begin{aligned}
a_{0}=\frac{b_{0}\left(a-1 b_{1}+a_{1}\right) \pm\left(a-1 b_{1}-a_{1}\right)}{\sqrt{ }} \sqrt{b_{0}^{2}-4 b_{1}} & \text { Then, } \\
2 b_{1} & \frac{a_{-1} \mathrm{e}^{-\xi}+a_{0}+a_{1} \mathrm{e}^{\xi}}{\mathrm{e}^{-\xi}+b_{0}+b_{1} \mathrm{e}^{\xi}}
\end{aligned}
$$

is a solution of ODE (2.1) with

$$
\begin{aligned}
& q_{0}=\frac{a_{-1}^{2} a^{2}}{\left(b_{1} a_{-1}-a_{1}\right)^{2},} \\
& q_{1}=\frac{-\quad a_{-1}-2 b_{1} a^{2} a_{1}}{\left(b_{1} a-1-a_{1}\right)^{2}}, \\
& q_{2}=\frac{a^{2}+4 a-1 b_{1} a_{1}+a_{-1}^{2} b^{2}}{\left(b_{1} a-1-a_{1}\right)^{2}}, \\
& q_{3}=\frac{-2 a_{1} b_{1}-2 a-1 b_{2}^{2}}{\left(b_{1} a-1-a_{1}\right)}, \\
& q_{4}=\frac{b_{1}^{2}}{\left(b_{1} a-1-a_{1}\right)^{2}} .
\end{aligned}
$$

Note that additional solutions of ODE (2.1) can be constructed using Weierstrass' elliptic function. The reader is directed to [17] for more details.

\section{A generalized expansion method}

We will briefly outline a generalized expansion method for constructing traveling wave solutions. Similar procedures have been developed in references [2, 3]. However, the new results given in the previous section ensure that our method yields many new solutions when applied to some classical partial differential equations. This will be clearly demonstrated in Sections 4-6.

We consider the following non-linear wave equation:

$$
H\left(u, u_{t}, u_{x}, u_{t t}, u_{x x}, u_{x t}, \ldots\right)=\mathrm{o}
$$

where $u:=u(x, t)$ is a real or complex-valued function, $H$ is a given function involving powers of its arguments and the subscripts denote differentiation. We will consider 
candidate traveling wave solutions that take the form

$$
u(x, t)=\tilde{u}(\xi)={ }_{j=0}^{N} c_{j}[F(\xi)]^{j}
$$

where $\xi=\mu(x-v t), \mu>0$ is the wave number, $v$ is the traveling wave velocity, $N$ is an integer, $F$ is a non-trival solution of ODE (2.1) with coefficients $q_{k}, k=0, \ldots, 4$, and $c_{j}, j=0, \ldots, N$, are constants with $c_{N} /=0$. Depending on the form of $H, \mu$ and $v$ will be determined or remain as free parameters.

Note that $\tilde{u}$ given by (3.2) is a polynomial function of $F$. Hence, it is readily seen that, for each integer $k \geq 1, \tilde{u}^{k}$ is also a polynomial in $F$. In this case, we use the degree notation $O(\cdot)$ to denote the index of the highest power of $F$. Thus,

$$
O\left(\tilde{u}^{K}\right)=N \kappa, \quad K \geq 1 .
$$

The derivatives of $F$ can be obtained by repeatedly differentiating both sides of (2.1). For example,

$$
\begin{aligned}
& F^{11}=\frac{q_{1}}{2}+q_{2} F+\frac{3 q_{3}}{2} F^{2}+2 q_{4} F^{3} \text {, } \\
& 111=\left(q_{2}+3 q_{3} F+6 q_{4} F^{2}\right) F^{1}, \\
& F F^{1111}=\left(3 q_{0} q_{3}+{ }_{z}^{1} q_{1} q_{2}\right)+\left(q_{2}^{2}+{ }_{z}^{9} q_{1} q_{3}+12 q_{0} q_{4}\right) F \\
& +15\left({ }_{2} q_{2} q_{3}+q_{1} q_{4}\right) F^{2}+\left(20 q_{2} q_{4}+\frac{{ }_{2}}{2} q_{3}^{2}\right) F^{3}+30 q_{3} q_{4} F^{4}+24 q_{4}^{2} F^{5} \text {. }
\end{aligned}
$$

It is not difficult to show that only the even derivatives are polynomials in $F$. The odd derivatives also contain terms of the form $F^{j}\left(F^{1}\right)$, where $j$ is a non-negative integer. In this case, we define $O\left(F^{1}\right)=2$ and so

$$
O\left(F^{j}\left(F^{1}\right)\right)=j+2, \quad j \geq 0 .
$$


By differentiating (3.2), we can also deduce the derivatives of $\tilde{u}$. For example,

$$
\begin{aligned}
\tilde{u}^{1}= & \left(c_{1}+\ldots+N c_{N} F^{N-1}\right) F^{1} \\
\tilde{u}^{11}= & \left(c_{1}+\ldots+N c_{N} F^{N-1}\right) F^{11}+\left[2 c_{2}+\ldots+N(N-1) c_{N} F^{N-2}\right]\left(F^{1}\right)^{2} \\
\tilde{u}^{111}= & \left(c_{1}+\ldots+N c_{N} F^{N-1}\right) F^{111}+3\left[2 c_{2}+\ldots+N(N-1) c_{N} F^{N-2}\right] F^{1} F^{11} \\
& +\left[6 c_{3}+\ldots+N(N-1)(N-2) c_{N} F^{N-3}\right]\left(F^{1}\right)^{3} \\
\tilde{u}^{1111}= & \left(c_{1}+\ldots+N c_{N} F^{N-1}\right) F^{1111} \\
& +4\left[2 c_{2}+\ldots+N(N-1) c_{N} F_{N-2}\right] F^{1} F^{111} \\
& +3\left[2 c_{2}+\ldots+N(N-1) c_{N} F^{N-2}\right]\left(F^{11}\right)^{2} \\
& +6\left[6 c_{3}+\ldots+N(N-1)(N-2) c_{N} F^{N-3}\right]\left(F^{1}\right)^{2} F^{11} \\
& +\left[24 c_{4}+\ldots+N(N-1)(N-2)(N-3) c_{N} F^{N-4}\right]\left(F^{1}\right)^{4}
\end{aligned}
$$

where the derivatives of $F$ are given in (2.1) and (3.4). Higher order derivatives can be obtained similarly. Again, only the even derivatives of $\tilde{u}$ are polynomials in $F$. It is readily seen that

$$
O\left(\tilde{u}^{(K)}\right)=N+K, \quad K \geq 1
$$

When $\tilde{u}$ is substituted into (3.1), the original partial differential equation in $x$ and $t$ is reduced to a non-linear ODE in $\xi$. We will normally choose $N$ so that the degrees of the highest order derivative term and highest order non-linear term in this reduced ODE are balanced. However, this does not always result in an integral value for $N$. In this case, it is sometimes possible to proceed by letting $\tilde{u}=V^{\frac{1}{\tau}}$, where $T$ is the denominator of the fractional value of $N$ (assuming the denominator and numerator have no common factors), and solving the resulting equation for $v$. This is illustrated in the following example.

Example 1. Consider the following Boussinesq-type equation:

$$
u_{t t}-u_{x x}+u_{x x x x}+\left(u^{5}-u^{3}\right)_{x x}=0
$$


By letting $u(x, t)=\tilde{u}(\mu(x-v t))$, the above partial differential equation is reduced to the following ODE:

$$
v^{2} \tilde{u}^{11}-\tilde{u}^{11}+\mu^{2} \tilde{u}^{1111}+\left(\tilde{u}^{5}-\tilde{u}^{3}\right)^{11}=0 .
$$

Integrating twice yields

$$
v^{2} \tilde{u}-\tilde{u}+\mu^{2} \tilde{u}^{11}+\tilde{u}^{5}-\tilde{u}^{3}=0 .
$$

Here, the highest order non-linear term is $\tilde{u}^{5}$, and the highest order derivative term is $\tilde{u}^{11}$. Balancing these two terms using (3.3) and (3.6) gives $5 N=N+2$, or $N={ }_{\overrightarrow{2}}$. Setting $\tilde{u}=v^{\frac{1}{2}},(3.7)$ becomes

$$
\left(v^{2}-1\right) v^{2}+\frac{\mu_{2}}{4}\left[2 v v^{11}-\left(v^{1}\right)^{2}\right]+v^{4}-v^{3}=0 .
$$

Now, we can balance $\left(v^{1}\right)^{2}$ and $v^{4}$ to yield $N=1$. Hence, we can search for traveling wave solutions of (3.8) which take the form $v(\mu(x-v t))=c_{0}+c_{1} F(\mu(x-v t))$, for constants $c_{0}$ and $c_{1}$. If such a $v$ can be determined, then it is easy to derive $\tilde{u}$. •

It is noted in Example 1 that substituting $\tilde{u}$ into (3.1) yields a non-linear ODE in $\xi$. When the derivatives of $\tilde{u}$ are substituted into this reduced ODE, we will obtain a linear combination of $F^{j}\left(F^{1}\right)^{k}$, where $j \geq 0$ is an integer and $k \in\{0,1\}$. If $v, \mu$, and $c_{j}, j=0, \ldots, N$, and $q_{k}, k=0, \ldots, 4$, can be chosen to make each coefficient in this linear combination zero, then the resulting $\tilde{u}$ will satisfy the original partial differential equation (3.1). However, in this procedure, we sometimes end up with $c_{j}=0$, $j=0, \ldots, N$ (we encounter this in Section 6). In this case, we can use the following alternative solution form proposed in [2]:

$$
\tilde{u}(\xi)=c_{0,0}+{ }_{j=1}^{N} \underset{-1, j}{c}[F(\xi)]^{j}+c_{2, j}[F(\xi)]^{j-1} F^{1}(\xi),
$$


where $c_{0,0}, c_{k, j}, k=1,2, j=1, \ldots, N$, and $\theta$ are constants.

Notice that each of the Jacobian elliptic solutions of ODE (2.1) reported in $[2,13,14]$ can be written as a scalar multiple of some $\phi_{j, k}(\cdot, 0), j \in\{1, \ldots, 6\}, k \in\{1, \ldots, 4\}$. Hence, by applying our expansion method with (3.2) and Theorem 1 to a non-linear partial differential equation, we can replicate every Jacobian elliptic solution obtained using the methods presented in [13,14]. Applying our expansion method with (3.9) and Theorem 1 to a non-linear partial differential equation, we can obtain all Jacobian elliptic solutions obtained using the method presented in [2]. Similarly, each Jacobian elliptic solution of ODE (2.1) reported in $[3,4]$ with $\omega=1$ can be written as a scalar multiple of some $\phi_{j, k}(\cdot, 0), j \in\{1, \ldots, 6\}, k \in\{1, \ldots, 4\}$. It is also evident that, for the special case $\theta=0$, using our expansion method with (3.9) and Theorems 1 and 2, we

can recover every Jacobian elliptic solution obtained using the method of [3,21]. Hence, by virtue of the new results in Section 2, our method is a significant generalization of the work reported in $[2,3,13,14]$.

\section{Traveling wave solutions for the Boussinesq equa- tion}

Consider the well-known Boussinesq equation

$$
u_{t t}=u_{x x}+u_{x x x x}+3\left(u^{2}\right)
$$

where $u:=u(x, t)$ is a real-valued function. Various methods have been used to solve Boussinesq type equations $[7,8,11]$. Here, the general expansion method will be used to derive new traveling wave solutions for (4.1). Letting $u(x, t)=\tilde{u}(\xi)$, where $\xi$ is as defined in Section 3, (4.1) becomes the following ODE

$$
v^{2} \tilde{u}^{11}=\tilde{u}^{11}+\mu^{2} \tilde{u}^{1111}+3\left(\tilde{u}^{2}\right) \text { 11 } .
$$


Balancing $\left(\tilde{u}^{2}\right)^{11}$ and $\tilde{u}^{1111}$ gives $2 N+2=N+4$, or $N=2$. Hence, we will search for traveling wave solutions of the form

$$
\tilde{u}(\xi)=c_{0}+c_{1} F(\xi)+c_{2}[F(\xi)]^{2}
$$

where $c_{2} /=0$ and $F$ satisfies $\operatorname{ODE}(2.1)$ with coefficients $q_{k}, k=0, \ldots, 4$. Substituting (4.3) into (4.2) and using (2.1) and (3.4)-(3.5), we obtain the following sufficient conditions for $\tilde{u}$ to satisfy (4.1):

$$
\begin{aligned}
& c_{0}=\frac{3 \mu^{2} q^{2}-16 \mu^{2} q q_{2}-4 q_{4}+4 q_{4} v^{2}}{24 q_{4}} \\
& c_{1}=-\mu q_{3}, \\
& c_{2}=-2 \mu^{2} q_{4}, \\
& q_{1}=\frac{q_{3}\left(4 q_{2} q_{4}-q_{3}^{2}\right)}{8 q_{4}^{2}} .
\end{aligned}
$$

That is, if a solution $F$ of ODE (2.1) with coefficients satisfying $q_{1}=\frac{q_{3}\left(4 q_{2} q_{4}-q_{3}^{2}\right)}{8 q_{4}^{2}}$ and $q_{4} /=0$ can be found, then

$$
\tilde{u}(\xi)=\frac{3 \mu^{2} q_{3}^{2}-16 \mu^{2} q_{4} q_{2}-4 q_{4}+4 q_{4} v^{2}}{24 q_{4}}-\mu^{2} q_{3} F(\xi)-2 \mu^{2} q_{4}[F(\xi)]^{2}
$$

is a solution of the Boussinesq equation (4.1). Now, we generalize this solution form further. Note that, if $q_{1}=q_{3}=0$, then (4.5) reduces to

$$
\tilde{u}(\xi)=\frac{v^{2}-1-4 \mu^{2} q_{2}}{6}-2 \mu^{2} q_{4}[F(\xi)]^{2}
$$

If $q_{0} /=0$, then using Theorem 2 with (4.6) gives the following solution form for equation (4.1):

$$
\tilde{u}(\xi)=\frac{v^{2}-1-4 \mu^{2} q_{2}}{6}-2 \mu^{2} q_{4}[F(\xi)]^{2}-\frac{2 \mu^{2} q_{0}}{[F(\xi)]^{2}}
$$


Furthermore, note that (4.5) can be rewritten as

$$
\tilde{u}(\xi)=\frac{\mu^{2} q_{3}^{2}}{4 q_{4}}+\frac{v^{2}-4 \mu^{2} q_{2}-1}{6}-2 \mu^{2} q_{4}\left(F(\xi)+\frac{q_{3}}{4 q_{4}}{ }^{2} .\right.
$$

The solution forms (4.7) and (4.8) provide motivation for the following, more general, candidate traveling wave solution:

$$
\tilde{u}(\xi)=\frac{\mu^{2} q_{3}^{2}}{4 q_{4}}+\frac{v^{2}-4 \mu 2 q_{2}-1}{6}-2 \mu^{2} q_{4}\left(F(\xi)+{\frac{q_{3}}{4 q_{4}}}^{2}+d F(\xi)+{\frac{q_{3}}{4 q_{4}}}^{-2}\right. \text {, }
$$

where $d$ is a constant. By substituting (4.9) into (4.2), the value of $d$ can be determined. We summarize our results in the form of the following theorem.

Theorem 6. For each $j=1,2$, let $\varepsilon_{j} \in\{0,1\}$. Suppose that $F$ is a solution of $\operatorname{ODE}(2.1)$ with coefficients $q_{j}, j=0, \ldots, 4$, satisfying $q_{4} /=0$ and $q_{1} \frac{q_{3}\left(4 q_{2} q_{4}-q_{3}^{2}\right)}{8 q_{4}^{2}}$. $=$

Then, for any $\mu$ and $v$,

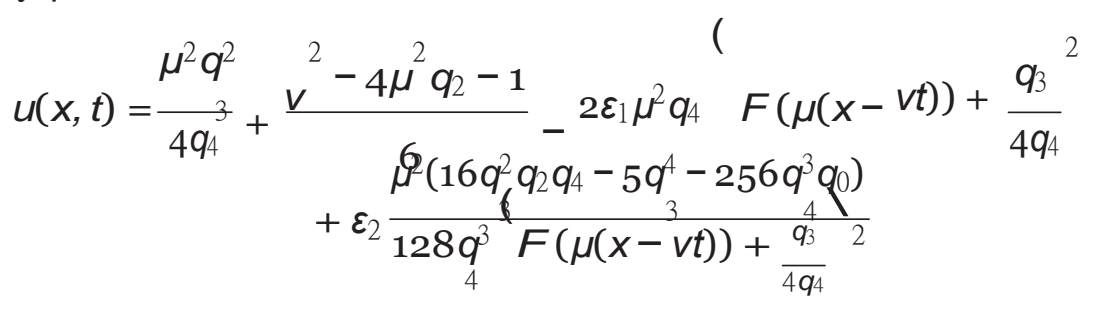

is a solution of the Boussinesq equation (4.1).

Remark 3. Note that the solution form given in Theorem 6 includes both (4.5) and (4.7) as special cases.

Notice that the coefficients of ODE (2.1) in Theorem 5 satisfy the requirements of Theorem 6. Thus, we can apply Theorem 6 with the solutions reported in Theorem 5 to obtain the following class of traveling wave solutions for the Boussinesq equa- 
tion (4.1):

$$
\begin{aligned}
u_{1}(x, t)=\frac{v^{2}-1+2 \mu^{2}}{6}-\varepsilon_{1} \frac{\mu^{2}}{2} & \frac{\left(\mathrm{e}^{-\mu(x-v t)} \pm{ }^{V} \lambda^{2}-4 \vartheta+\vartheta \mathrm{e}^{\mu(x-v t)}\right.}{2} \\
-\frac{\varepsilon_{2}}{2} \frac{\mu_{2}}{2} & \frac{\mathrm{e}^{-\mu(x-v t)}+\lambda+\vartheta \mathrm{e}^{\mu(x-v t)}}{-\mathrm{e}^{-\mu(x-v t)} \pm \lambda^{2}-4 \vartheta+\vartheta \mathrm{e}^{\mu(x-v t)}}
\end{aligned},
$$

where, for each $j=1,2, \varepsilon_{j} \in\{0,1\}$, and $\lambda, \vartheta, \mu$ and $v$ are arbitrary real constants such that $\vartheta \diamond \lambda^{2} / 4$.

It should be addressed here that the above class of solutions includes all of those obtained by combining Theorems 4 and 6 . Note that, for some cases, the denominators in the expression of $u_{1}$ can be equal to zero at certain points, and thus, such a solution is unbounded. For example, $u_{1}$ with $\varepsilon_{1}=\varepsilon_{2}=1$ and $\vartheta /=0$ is unbounded. It is also noted that, for some cases, the solution $u_{1}$ is bounded. For instance, $u_{1}$ with $\varepsilon_{1}=1$, $\varepsilon_{2}=0,0 \vartheta \vartheta \lambda^{2} / 4$ and $\lambda$ ): 0 is bounded. For the bounded case, clearly, the solution $u_{1}$ gives a single wave that moves in the $x$-direction with velocity $v$ and as $\mu(x-v t) \rightarrow \pm \infty, u_{1}(x, t) \rightarrow\left(v^{2}-1+2 \mu^{2}\right) / 6-\mu^{2}\left(\varepsilon_{1}+\varepsilon_{2}\right) / 2$.

Choosing $\vartheta=1$ and replacing $\lambda$ by $2 \lambda, u_{1}$ becomes

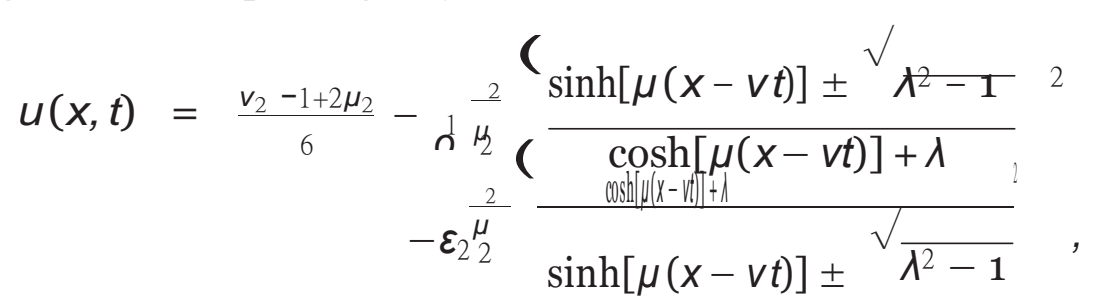

where, for each $j=1,2, \varepsilon_{j} \in\{0,1\}$, and $\lambda, \mu$ and $v$ are arbitrary real constants such that $\lambda \geq 1$ or $\lambda \leq-1$.

Since $u$ is a real-valued function, the arbitrary constants in $u_{1}$ are generally real. However, this is actually an unnecessary restriction - these constants can be complex provided that $u_{1}$ remains real. If $\mu$ is replaced by $\mathrm{i} \mu$ in (4.10), then we obtain another 
class of solutions:

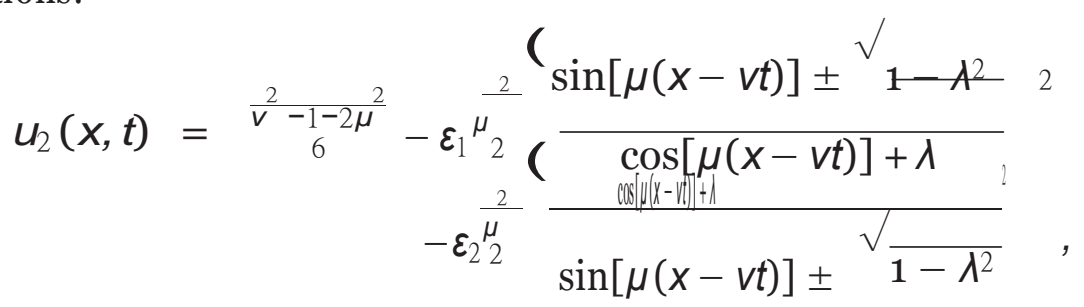

where, for each $j=1,2, \varepsilon_{j} \in\{0,1\}$, and $\mu, v$ and $\lambda$ are arbitrary real constants such that $-1 \leq \lambda \leq 1$. Obviously, the solution $u_{2}$ is unbounded.

Remark 4. In [19], the solutions of (4.1) were obtained using the $\sinh / \cosh$ ansatz III method, the sinh-cosh ansatz III method, the tanh method and the sine-cosine method. Each of these solutions is a special case of $u_{1}$ or $u_{2}$.

To apply Theorem 1 in conjunction with Theorem 6, we must choose $\gamma$ so that the hypotheses of Theorem 6 are satisfied. That is, $\gamma$ should be chosen so that the following equation holds:

$$
p_{j, 1}(\gamma)=\frac{p_{j, 3}(\gamma)\left(4 p_{j, 2}(\gamma) p_{j, 4}(\gamma)-\left[p_{j, 3}(\gamma)\right]^{2}\right)}{8\left[p_{j, 4}(\gamma)\right]^{2}} .
$$

Then, the corresponding solutions reported in Theorem 1 can be used with Theorem 6. Applying this procedure, we can obtain periodic solutions of Boussinesq equation (4.1) in terms of Jacobi elliptic functions. For each $j=1, \ldots, 12$, choosing $q_{k}=p_{j, k}$, $k=0, \ldots, 4$, and employing Theorem 6 give solutions $u_{j}, j=3, \ldots, 26$. Some of the solutions are listed below.

$$
\begin{aligned}
& u_{3}(x, t)=\frac{v^{2}-1+4 \mu^{2}\left(m^{2}+1\right)}{v^{2}-1+4 \sigma_{1}\left(m^{2}-2\right)}-2 \mu{ }^{2} r^{I} \varepsilon_{1} m^{2} \operatorname{sn}^{2}(\xi)+\varepsilon_{2} \operatorname{sn}^{-}{ }^{2}(\xi), \\
& u_{4}(x, t)=\frac{v^{2}-1+4 \mu^{2}\left(m^{2}-2\right)}{6}+2 \mu^{2} \varepsilon_{1} \mathrm{dn}^{2}(\xi)+\frac{1-m^{2}}{\mathrm{dn}^{2}(\xi)} \text {, }
\end{aligned}
$$

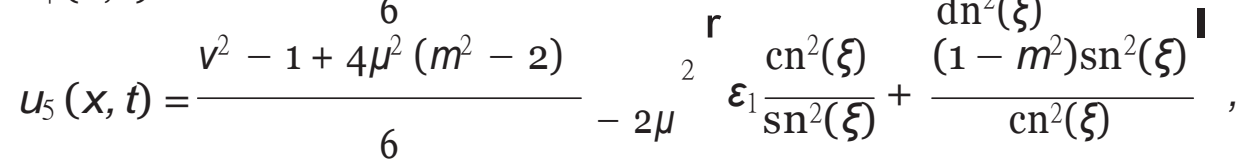




$$
\begin{aligned}
& u_{6}(x, t)=\frac{v^{2}-1+4 \mu^{2}\left(m^{2}+1\right)}{6}-2 \mu{ }^{2} m^{2} \frac{\mathrm{cn}^{2}(\xi)}{\mathrm{dn}^{2}(\xi)}+\frac{\mathrm{dn}^{2}(\xi)}{\mathrm{cn}^{2}(\xi)},
\end{aligned}
$$

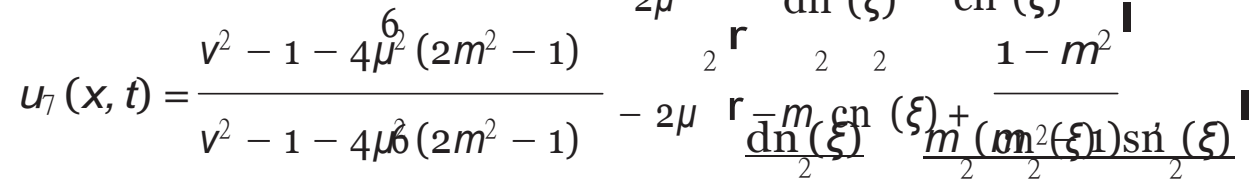

$$
\begin{aligned}
& u_{8}(x, t)=\frac{}{6}-2 \mu^{2} \operatorname{sn}^{2}(\xi)+\operatorname{dn}^{2}(\xi) \\
& u_{9}(x, t)=\frac{v^{2}-1+2 \mu^{2}\left(2 m^{2}-1\right)}{6}-\frac{\mu^{2}[1-\operatorname{cn}(\xi)]}{2[1+\operatorname{cn}(\xi)]} \text {, }
\end{aligned}
$$

where, for each $j=1,2, \varepsilon_{j} \in\{0,1\}$, and $\mu$ and $v$ are arbitrary real constants. The other Jacobi elliptic function solutions are listed in the Appendix.

Remark 5. It follows from Remark 1 that $u_{j}, j=3, \ldots, 26$, still satisfy the Boussinesq equation (4.1) even if $\operatorname{cn}(\xi), \operatorname{sn}(\xi)$ and $\operatorname{dn}(\xi)$ are replaced, respectively, by $\pm \mathrm{cn}(\xi)$, $\pm \operatorname{sn}(\xi)$ and $\pm \operatorname{dn}(\xi)$.

Remark 6. It is interesting to note that, for each $j \in\{3, \ldots, 26\}$, the solution $u_{j}$ becomes a special case of $u_{1}$ as $m \rightarrow 1$. Similarly, as $m \rightarrow 0, u_{j}$ becomes a special case of $u_{2}$.

Remark 7.The solution $U_{3}$ is identical to the solutions reported in [12, 22], and the solution $u_{9}$ is the same as the solution reported in [23] (for $\varepsilon^{2}=1, \alpha=1$ and $\beta=3$ ). However, all of the other Jacobian elliptic function solutions are new solutions. Furthermore, if the candidate traveling wave solutions of the form (3.9) are considered and our new results in Section 2 are applied, then many additional solutions can be obtained.

To show the physical insight of these solutions, here we take $u_{4}$ and $u_{7}$ as examples. Figure 1 shows the wave profile of the solution $u_{4}$ with $m=0.99, \mu=1$ and $v=-1$. Clearly, the solution is a periodic function describing the traveling of waves 

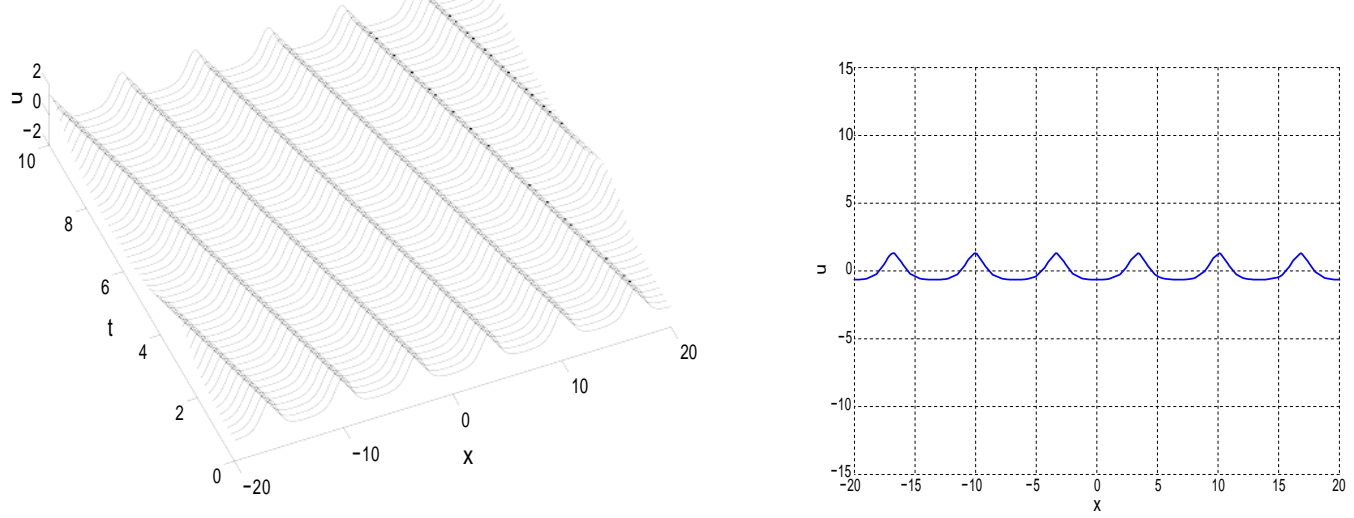

Figure 1: The plot of the solution $u_{4}$ to the Boussinesq equaiton (4.1) with $m=0.99$, $\mu=1$ and $v=-1$ and the initial status of $u_{4}$.

in the negative $x$-direction. Figure 2 shows the graph of the solution $u_{7}$ for $m=0.9$, $\mu=1$ and $v=-2$. Note that $u_{7}$ becomes infinity when $\operatorname{cn}(\mu(x-v t), m)=0$, that is, $\mu(x-v t)=(2 n+1) K$, where $K={ }_{0}^{f_{\pi / 2}}\left(1-m^{2} \sin ^{2}(s)^{)_{-1 / 2}} d s\right.$ and $n=0, \pm 1, \ldots$ For instance, in Figure 2, $u_{7}$ becomes negative infinity when the point $(x, t)$ is close to the lines $x+2 t=2.280549138(2 n+1)$, where $n=0, \pm 1, \ldots$ It is also noted from the expression of the solutions $u_{3}$ with $\varepsilon_{2}=1, u_{5}, \ldots, u_{9}$ that these solutions are unbounded, since the denominator in the expression can be zero at certain points.

\section{Traveling wave solutions for the modified KdV equation}

We consider the following modified KdV equation:

$$
u_{t}+u^{2} u_{x}+u_{x x x}=0
$$



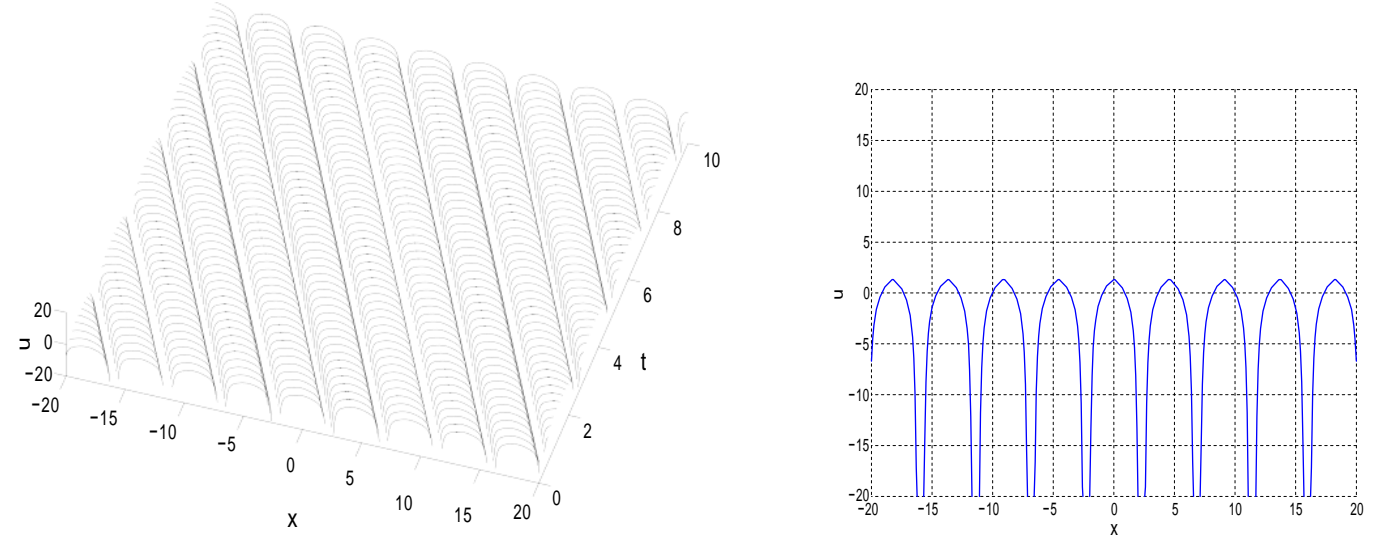

Figure 2: The plot of the solution $u_{7}$ to the Boussinesq equaiton (4.1) with $m=0.9$, $\mu=1$ and $v=-2$ and the initial status of $u_{7}$.

where $u:=u(x, t)$ is a complex-valued function. Letting $u(x, t)=\tilde{u}(\xi)$, where $\xi$ is as defined in Section 3, (5.1) is reduced to the ODE

$$
-v \tilde{u}^{1}+\tilde{u}^{2} \tilde{u}^{1}+\mu^{2} \tilde{u}^{111}=0
$$

Balancing $\tilde{u}^{2} \tilde{u}^{1}$ and $\tilde{u}^{111}$ yields $N=1$. Thus, we now consider candidate traveling wave solutions of the form

$$
\tilde{u}(\xi)=c_{0}+c_{1} F(\xi)
$$

where $c_{1} /=0$, and $F$ satisfies ODE (2.1) with coefficients $q_{k}, k=0, \ldots, 4$. Substituting $\tilde{u}$ into (5.2), we obtain the following sufficient conditions for $\tilde{u}$ to satisfy (5.2):

$$
\begin{aligned}
& c^{2}+6 \mu^{2} q_{4}=0, \\
& 2 c_{0} c_{1}+3 \mu^{2} q_{3}=0, \\
& -v+c_{0}^{2}+\mu^{2} q_{2}=0 .
\end{aligned}
$$


According to (5.3),

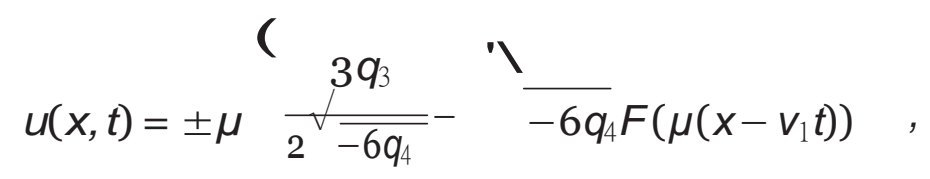

where $v_{1}=\mu^{2} q_{2}-\frac{3 q_{3}{ }^{2}}{8 q_{4}}$ and $\mu$ is an arbitrary constant, is a solution of (5.1). Now, if $q_{1}=q_{3}=0$ and $q_{0} /=0$, then Theorem 2 can be applied with (5.4) to give the following solution form of (5.1):

$$
u(x, t)=j_{1} \mu_{-}^{\prime-} 6 q_{0} \quad j_{2} \underset{q_{0}}{-} F\left(\mu\left(x-v_{2} t\right)\right)+\frac{1}{F\left(\mu\left(x-v_{2} t\right)\right)},
$$

where $j_{j}= \pm 1, j=1,2, v_{2}=\mu^{2}{ }^{(} q_{2}-j_{2} 6 q_{0}{ }_{q_{4}} \frac{1}{q_{0}}$ and $\mu$ is an arbitrary constant. In addition, if $q_{4}=\frac{q_{0} q_{2}}{q_{1}^{2}}$ and $q_{0} q_{1} /=0$, then Theorem 3 can be applied with (5.4) to yield another solution form of (5.1):

$$
u(x, t)= \pm \mu \frac{3 q_{1}}{\sqrt{\frac{-6 q_{0}}{r}}}-\frac{\mathbf{1}}{-6 q_{0}}{ }_{\frac{q_{3}}{q_{1}}} F\left(\mu\left(x-v_{3} t\right)\right)+\frac{\mathbf{I}}{F\left(\mu\left(x-v_{3} t\right)\right)}
$$

where $v_{3}=\mu^{2} q_{2}-\frac{6 q_{0} q_{3}}{q_{1}}-\frac{3 q_{2}}{8 q_{0}}$ and $\mu$ is an arbitrary constant.

We can apply Theorem 4 with (5.4) to obtain the following class of traveling wave solutions of (5.1):

$$
u_{1}(x, t)=\lambda+\frac{\frac{3 \vartheta \mu^{2}}{\lambda}}{\mathrm{e}^{-\mu\left(x-\left(\mu^{2}+\lambda^{2}\right) t\right)}+\vartheta+\frac{\vartheta^{2}\left(2 \lambda^{2}+3 \mu^{2}\right)}{8 \lambda^{2}} \mathrm{e}^{\mu\left(x-\left(\mu^{2}+\lambda^{2}\right) t\right)}},
$$

where $\lambda, \vartheta$ and $\mu$ are arbitrary parameters such that $\lambda /=0$. It is noted that, if $\lambda, \vartheta$ and $\mu$ are all real constants satisfying $\lambda \vartheta \mu /=0$, then $u_{1}$ describes a single wave travelling in the $x$-direction and $u_{1}(x, t) \rightarrow \lambda$, as $\mu\left(x-\left(\mu^{2}+\lambda^{2}\right) t\right) \rightarrow \pm \infty$.

We can also apply Theorem 5 with (5.6) to obtain another class of solutions of 
(5.1):

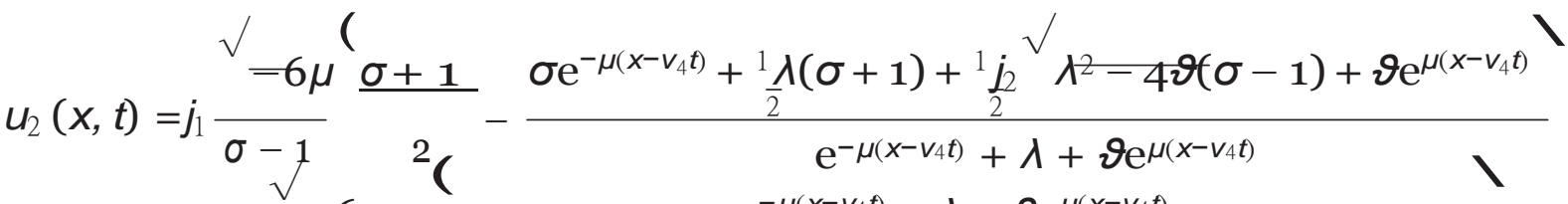

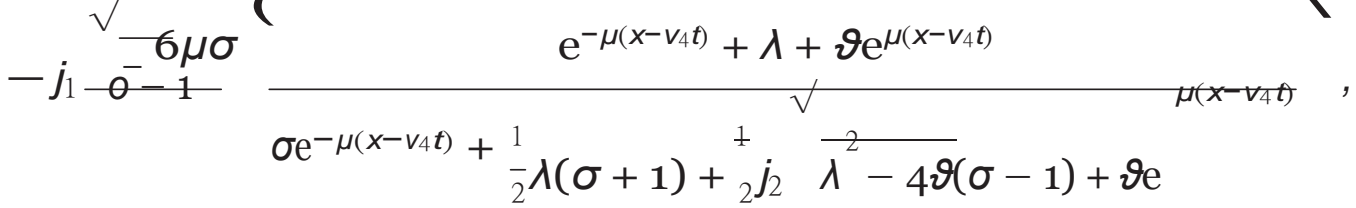

where $j_{j}= \pm \mathbf{1}, \boldsymbol{j}=\mathbf{1}, \mathbf{2}, \boldsymbol{v}_{4}=\frac{\stackrel{2}{2} \stackrel{2}{(\sigma+10 \sigma+1)}}{2(\sigma-1)^{2}}$, and $\lambda, \boldsymbol{\vartheta}$ and $\sigma$ are arbitrary constants such that $\sigma /=1$. Note that $u_{1}$ is the same as solution (18) in [6], obtained using the Exp-function method. However, $u_{2}$ is a new solution.

We also can obtain Jacobian elliptic solutions to the modified KdV equation (5.1) by combining Theorem 1 with (5.4)-(5.6).

1. For $k \in\{1, \ldots, 4\}, j \in\{1, \ldots, 12\}$ and $\gamma$ arbitrary, (5.4) with $F=\phi_{j, k}(\cdot, \gamma)$ and $q_{l}=p_{j,}(\gamma), l=0, \ldots, 4$, is a solution of (5.1).

2. For $k \in\{1, \ldots, 4\}$ and $j \in\{1,2,3,4,5,6,9,11\}$, (5.5) with $F=\phi_{j, k}(\cdot, 0)$ and $q_{l}=p_{j,(}(\mathrm{O}), l=0, \ldots, 4$, is a solution of $(5 \cdot 1)$.

3. For $k \in\{1, \ldots, 4\}$ and $j \in\{10,12\}$, (5.6) with $F=\phi_{j, k}(\cdot, 0)$ and $q_{l}=p_{j,}(0)$, $I=0, \ldots, 4$, is a solution of (5.1).

Thus, we can obtain many Jacobian elliptic solutions of (5.1). To keep the details to minimum, we will not list them all here. Instead, we just select some of them to compare our results with those reported in $[18,22]$. Note that our method can also be applied to the modified $\mathrm{KdV}$ equation considered in [18, 22].

Let $\gamma$ be such that $\gamma /= \pm 1$ and $\gamma /= \pm m$. Choosing $q_{k}=p_{2, k}(\gamma), k=0, \ldots, 4$, from 
(5.4), it follows that

$$
\begin{aligned}
& 3 \gamma\left(1+m^{2}-2 \gamma^{2}\right) \\
& u_{3}(x, t)=\mu \frac{}{-6\left(m^{2}-\gamma^{2}\right)\left(1-y^{2}\right)} \\
& 3 \gamma\left(1+m^{2}-2 \gamma^{2}\right) \\
& u_{4}(x, t)=\mu \frac{}{-6\left(m^{2}-y^{2}\right)\left(1-y^{2}\right)} \\
& 3 \gamma\left(1+m^{2}-2 \gamma^{2}\right) \\
& u_{5}(x, t)=\mu \frac{}{\left(\frac{3 \gamma\left(m^{2}-y^{2}\right)\left(1-y^{2}\right)}{3 \gamma\left(1+m^{2}-2 y^{2}\right)}\right.} \\
& 3 \gamma\left(1+m^{2}-2 \gamma^{2}\right) \\
& u_{6}(x, t)=\mu \\
& \frac{3 \gamma\left(1+m^{2}-2 \gamma^{2}\right)}{-6\left(m^{2}-\gamma^{2}\right)\left(1-\gamma^{2}\right)}
\end{aligned}
$$

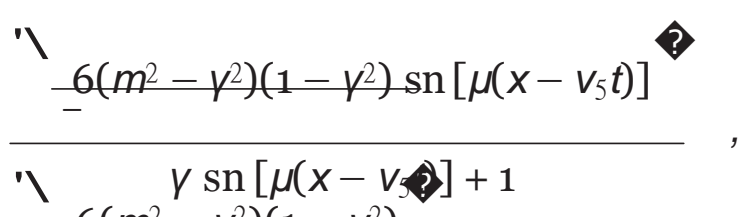

$$
\begin{aligned}
& 6\left(m^{2}-\gamma^{2}\right)\left(1-\gamma^{2}\right) \\
& \frac{-}{{ }^{\prime} \mathrm{x}+m \operatorname{sn}\left[\mu\left(x-v_{5} t\right)\right]} \text {, } \\
& 6\left(m^{2}-v^{2}\right)\left(1-v^{2}\right) \operatorname{dn}\left[\mu\left(x-v_{5} t\right)\right] \\
& \frac{-}{\prime} \operatorname{dn}\left[\mu\left(x-v_{5} t\right)\right]+m \operatorname{cn}\left[\mu\left(x-v_{5} t\right)\right] ? \\
& 6\left(m^{2}-v^{2}\right)\left(1-v^{2}\right) \in n\left[\mu\left(x-v_{5} t\right)\right] \\
& v \underset{\text { c }}{ }\left[\mu\left(x-v_{5} t\right)\right]+\operatorname{dn}\left[\mu\left(x-v_{5} t\right)\right] \text {, }
\end{aligned}
$$

where $v_{5}=\mu^{2}{ }_{6} \gamma^{2}-1-m^{2}-\frac{3 \gamma_{2}(1+m-2 \gamma)_{22}}{2\left(m^{2}-\gamma^{2}\right)\left(1-\gamma^{2}\right)}$ and $\mu$ is an arbitrary constant, are

solutions of (5.1). If $\gamma$ is any real number such that $m<|\gamma|<1$, then $u_{k}, k=3, \ldots, 6$, are real and bounded. Moreover, if $\gamma=0$, then according to (5.5), we can obtain the following two unbounded solutions:

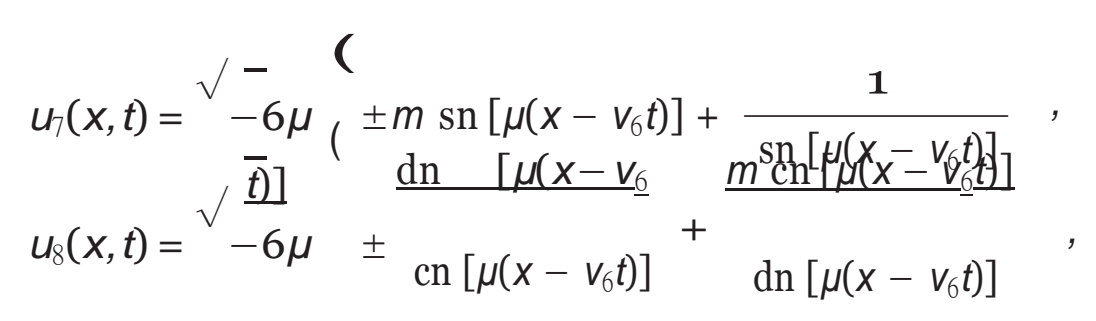

where $v_{6}=-\mu^{2}\left(1 \pm 6 m+m^{2}\right)$ and $\mu$ is an arbitrary constant.

Similarly, if $q_{k}=p_{3, k}(\gamma), k=0, \ldots, 4$, where $\gamma$ is an arbitrary constant such that $\underset{m}{V} /= \pm 1$ and $\gamma /= \pm \frac{V_{m^{2}}}{}$, then we get solutions of (5.1) as follows:

$$
\begin{aligned}
& u_{9}(x, t)=\mu \frac{3 \gamma\left(1-2 m^{2}-2 \gamma^{2}+2 \gamma^{2} m^{2}\right)}{-6\left(\gamma^{2} m^{2}-m^{2}-\gamma^{2}\right)\left(1-\gamma^{2}\right)} \\
& -\frac{{ }^{\prime}=6\left(\gamma^{2} m^{2}-m^{2}-\gamma^{2}\right)\left(1-\gamma^{2}\right) \operatorname{cn}\left[\mu\left(x-v_{7} t\right)\right]}{\gamma \operatorname{cn}\left[\mu\left(x-v_{7} t\right)\right]+1}, \\
& u_{10}(x, t)=\mu \frac{J \gamma\left(1-2 m^{2}-2 \gamma^{2}+2 \gamma^{2} m^{2}\right)}{-6\left(\gamma^{2} m^{2}-m^{2}-\gamma^{2}\right)\left(1-\gamma^{2}\right)} \\
& -\frac{6\left(\gamma^{2} m^{2}-m^{2}-\gamma^{2}\right)\left(1-\gamma^{2}\right)\left(1-m^{2}\right)}{\gamma \frac{m^{2}-1}{t}+m \operatorname{cn}\left[\mu\left(x-v_{7} t\right)\right]},
\end{aligned}
$$




$$
\begin{aligned}
& u_{11}(x, t)=\mu \frac{3}{\mathrm{~J}} \frac{3 \gamma\left(1-2 m^{2}-2 \gamma^{2}+2 \gamma^{2} m^{2}\right)}{-6\left(\gamma^{2} m^{2}-m^{2}-\gamma^{2}\right)\left(1-\gamma^{2}\right)} \\
& -\frac{{ }^{\prime}=6\left(\gamma^{2} m^{2}-m^{2}-\gamma^{2}\right)\left(1-\gamma^{2}\right) \operatorname{dn}\left[\mu\left(x-v_{7} t\right)\right]}{\gamma \operatorname{dn}\left[\mu\left(x-v_{7} t\right)\right]+\mathrm{i} m \operatorname{sn}\left[\mu\left(x-v_{7} t\right)\right]}, \\
& u_{12}(x, t)=\mu \frac{3 \gamma\left(1-2 m^{2}-2 \gamma^{2}+2 \gamma^{2} m^{2}\right)}{-6\left(\gamma^{2} m^{2}-m^{2}-\gamma^{2}\right)\left(1-\gamma^{2}\right)} \\
& -\frac{{ }^{\prime}=6\left(\gamma^{2} m^{2}-m^{2}-\gamma^{2}\right)\left(1-\gamma^{2}\right)\left(1-m^{2}\right) \operatorname{sn}\left[\mu\left(x-v_{7} t\right)\right]}{\gamma \sqrt{1-m^{2}} \operatorname{sn}\left[\mu\left(x-v_{7} t\right)\right]+\operatorname{dn}\left[\mu\left(x-v_{7} t\right)\right]},
\end{aligned}
$$

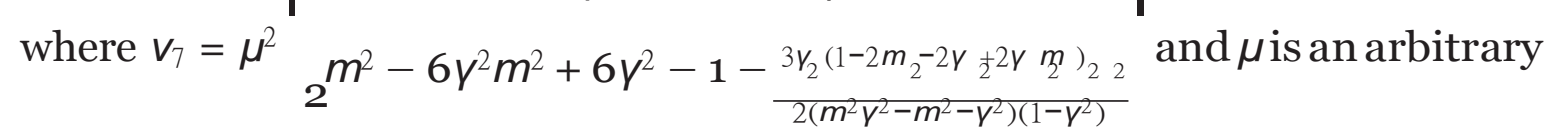
constant. Moreover, if $y=0$, then we have the unbounded solutions

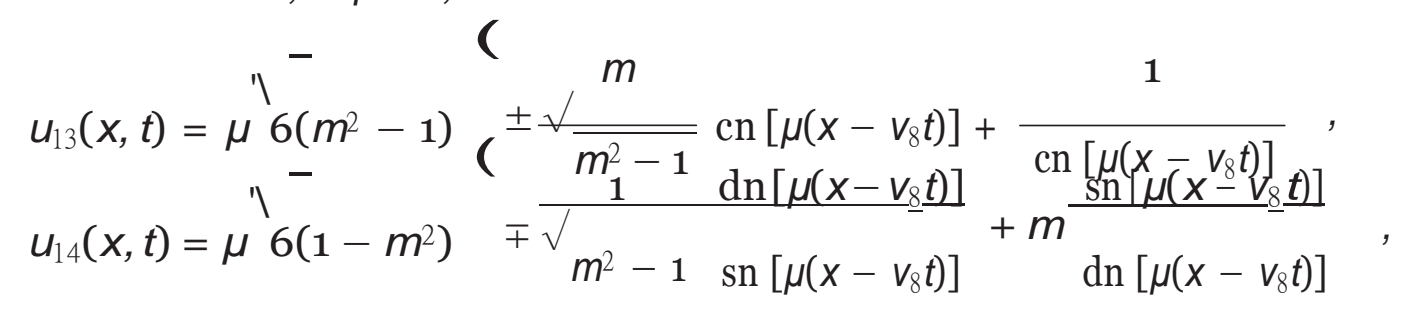

where $v_{8}=\mu^{2}\left(2 m^{2}-1 \pm 6 m^{\sqrt{ }} \overline{\left.m^{2}-1\right)}\right.$ and $\mu$ is an arbitrary constant.

If $q_{k}=p_{6, k}(\gamma), k=0, \ldots, 4$, where $\gamma$ is an arbitrary constant such that $m^{2} \gamma^{4}+$ $m^{2}+4 Y^{2}-2 m^{2} \gamma^{2} /=0$, then we can obtain another four solutions of (5.1)

$$
\begin{aligned}
& u_{15}(x, t)=\mu \frac{J}{J} \frac{3 \gamma\left(\gamma^{2} m^{2}-m^{2}+2\right)}{6\left(\gamma^{4} m^{2}+m_{\eta}^{2}+4 Y^{2}-2 \gamma^{2} m^{2}\right)} \\
& 6\left(\gamma^{4} m^{2}+m^{2}+4 Y^{2}-2 y^{2} m^{2}\right) \\
& -\overline{2 \gamma+\mathrm{i} 2 \operatorname{sn}\left[\mu\left(x-v_{9} t\right)\right]+2 \mathrm{cn}\left[\mu\left(x-v_{9} t\right)\right]} \text {, } \\
& u_{16}(x, t)=\mu+\frac{3 \gamma\left(\gamma^{2} m^{2}-m^{2}+2\right)}{6\left(\gamma^{4} m^{2}+m^{2}+4 \gamma^{2}-2 \gamma^{2} m^{2}\right)}
\end{aligned}
$$

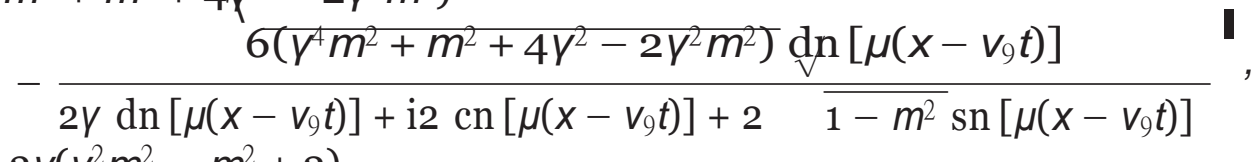

$$
\begin{aligned}
& u_{17}(x, t)=\mu \frac{\mathrm{J}}{+1} \frac{3 \gamma\left(\gamma^{2} m^{2}-m^{2}+2\right)}{6\left(\gamma^{4} m^{2}+m^{2}+4 Y^{2}-2 \gamma^{2} m^{2}\right)} \\
& -\frac{\sigma\left(\gamma^{4} m^{2}+m^{2}+4 \gamma^{2}-2 \gamma^{2} m^{2}\right) m \operatorname{sn}\left[\mu\left(x-v_{9} t\right)\right]}{2 \gamma m \operatorname{sn}\left[\mu\left(x-v_{9} t\right)\right]+\mathrm{i} 2+\mathrm{i} 2 \operatorname{dn}\left[\mu\left(x-v_{9} t\right)\right]},
\end{aligned}
$$




$$
\begin{aligned}
& u_{18}(x, t)=\mu \frac{\mathrm{J}}{\frac{\mathrm{J}}{6\left(\gamma^{4} m^{2}+\eta^{2}+4 Y^{2}-2 \gamma^{2} m^{2}\right)}} \\
& -\frac{\sigma\left(\gamma^{4} m^{2}+m^{2}+4 V^{2}-2 \gamma^{2} m^{2}\right) \operatorname{im} \operatorname{cn}\left[\mu\left(x+v_{9} t\right)\right]}{\text { i } 2 \gamma m \operatorname{cn}\left[\mu\left(x-v_{9} t\right)\right]+2 \ln \left[\mu\left(x-v_{9} t\right)\right]+2} \overline{1-m^{2}},
\end{aligned}
$$

where $v_{9}=\mu^{2} \frac{I_{2}-3 V_{2} m_{2}-2}{2}+\frac{3 \gamma_{2}\left(v_{2} m_{2}-m_{2}+2\right)}{2\left(m^{2} \gamma^{4}+m^{2}+4 \gamma^{2}-2 m^{2} \gamma^{2}\right)}$ and $\mu$ is an arbitrary constant.

Furthermore, choosing $\gamma=0$ yields that, for any $\mu$,

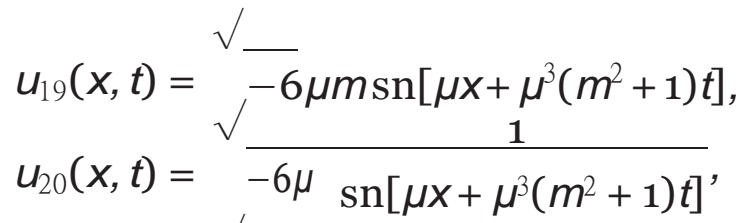

$$
\begin{aligned}
& u_{21}(x, t)=\sqrt{ }-6 \mu m \frac{\operatorname{cn}\left[\mu x+\mu^{3}\left(m^{2}+1\right) t\right]}{\operatorname{dn}\left[\mu x+\mu^{3}\left(m^{2}+1\right) t\right]}, \\
& u_{22}(x, t)={ }^{\sqrt{ }}-6 \mu \frac{\operatorname{dn}\left[\mu x+\mu^{3}\left(m^{2}+1\right) t\right]}{\operatorname{cn}\left[\mu x+\mu^{3}\left(m^{2}+1\right) t\right]}, \\
& u_{23}(x, t)=\overline{6} \mu m \operatorname{cn}\left[\mu x-\mu^{3}\left(2 m^{2}-1\right) t\right], \\
& u_{24}(x, t)=\mu \quad \overline{6\left(m^{2}-1\right)} \frac{1}{\operatorname{cn}\left[\mu x-\mu^{3}\left(2 m^{2}-1\right) t\right]}, \\
& u_{25}(x, t)=\mu^{\sqrt{ }-6} \frac{\operatorname{dn}\left[\mu x-\mu^{3}\left(2 m^{2}-1\right) t\right]}{\operatorname{sn}\left[\mu x-\mu^{3}\left(2 m^{2}-1\right) t\right]}, \\
& u_{26}(x, t)=\mu m^{\prime} \overline{6\left(1-m^{2}\right)} \frac{\operatorname{sn}\left[\mu x-\mu^{3}\left(2 m^{2}-1\right) t\right]}{\operatorname{dn}\left[\mu x-\mu^{3}\left(2 m^{2}-1\right) t\right]},
\end{aligned}
$$

are solutions of (5.1).

Remark 8. It follows from Remark 1 that $u_{j}, j=3, \ldots, 26$, still satisfy (5.1) even if $\operatorname{cn}(\cdot), \operatorname{sn}(\cdot)$ and $\operatorname{dn}(\cdot)$ are replaced, respectively, by $\pm \operatorname{cn}(\cdot), \pm \operatorname{sn}(\cdot)$ and $\pm \operatorname{dn}(\cdot)$.

Remark 9. If $y=0$, then $u_{3}, u_{9}$ and $u_{15}$ are the same as the solutions reported in [18] (with $a=1$ and $b=1$ ), and $u_{3}, u_{4}$ and $u_{7}$ are the same as those reported in [22] (for $\alpha=1$ and $\beta=1$ ). However, all of the other Jacobian elliptic solutions are new. More new solutions can be obtained if solution form (3.9) is used. 

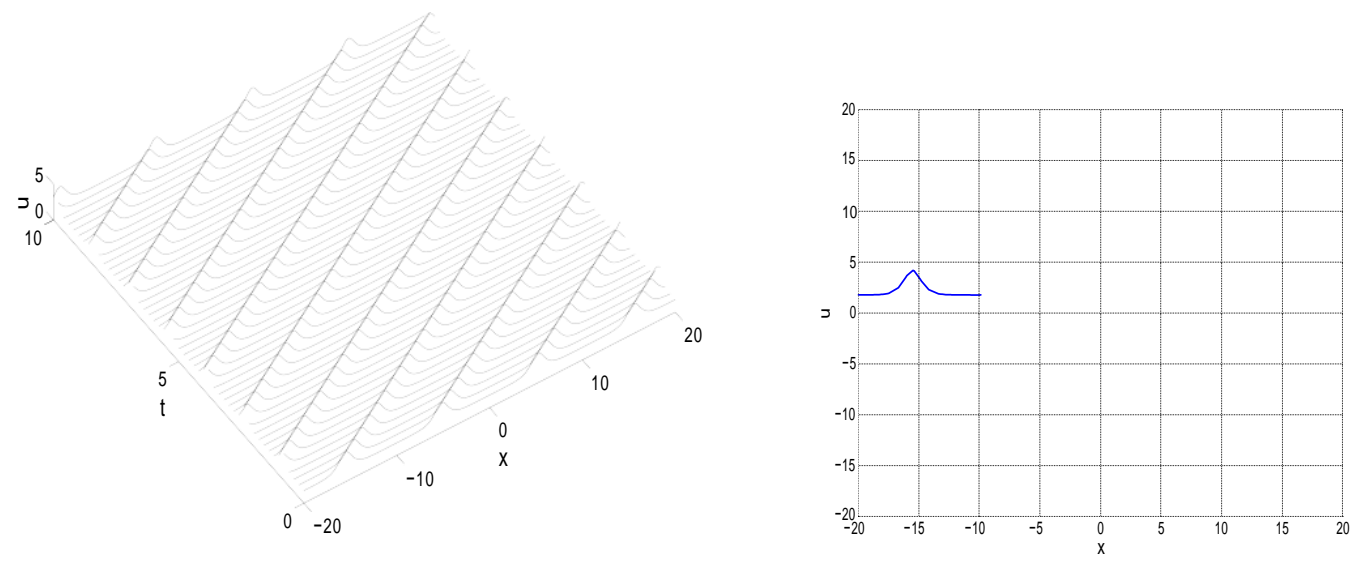

Figure 3: The plot of the solution $u_{6}$ to the modified KdV equation (5.1) with $m=0.95$, $\mu=1$ and $\gamma=0.96$ and the initial status of $u_{6}$.

To demonstrate the physical insight of the new solutions, we take $u_{6}$ as an example. By choosing $m=0.95$ and $\mu=1$, the wave profiles of the solution $u_{6}$ for two different values of $\gamma, \gamma=0.96$ and $\gamma=-0.96$, are displayed in Figures 3 and 4, respectively. Clearly, in both cases, the solutions describe the travelling of waves in the $x$-direction. Different values of $y$ yield different wave shapes.

\section{Traveling wave solutions for the shallow long wave approximate equations}

In this section, we will apply the method discussed in Section 3 to a system of partial differential equations. Consider the shallow long wave approximate equations

$$
\begin{aligned}
& u_{t}-u u_{x}-v_{x}+{ }^{1} t_{x x}=0, \\
& v_{t}-v u_{x}-u v_{x}-{ }^{1} \forall_{x x}=0,
\end{aligned}
$$

where $u:=u(x, t)$ is the horizontal velocity of water and $v:=v(x, t)$ is the height that deviates from the equilibrium position of the water. Substituting $u(x, t)=\tilde{u}(\xi)$ and 

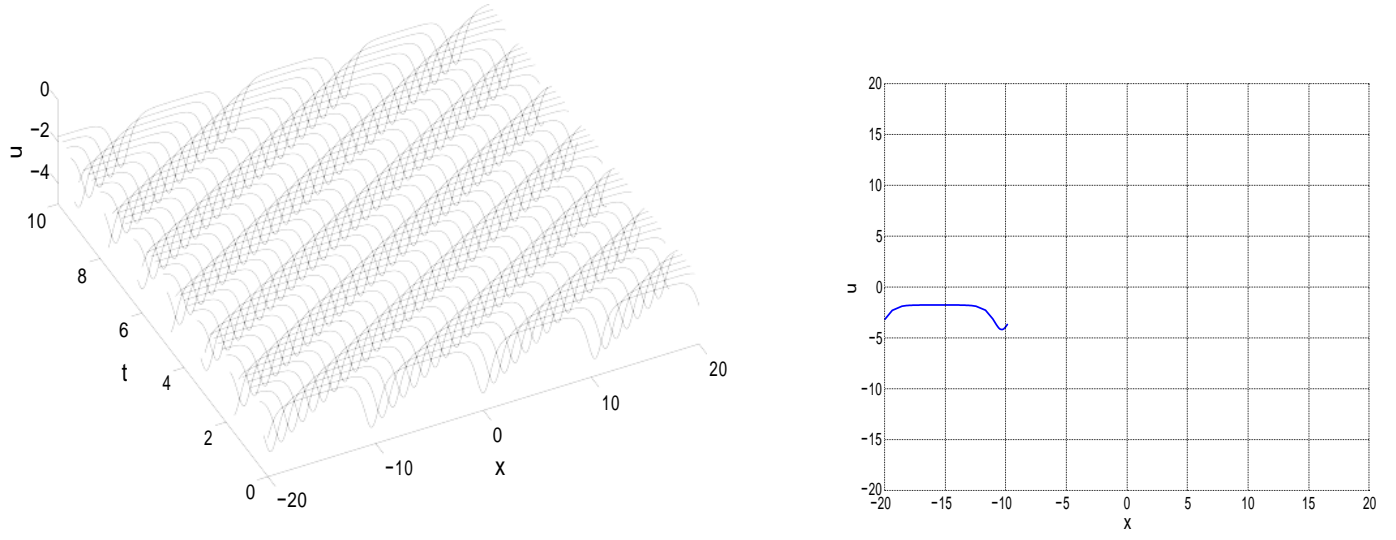

Figure 4: The plot of the solution $u_{6}$ to the modified KdV equation (5.1) with $m=0.95$, $\mu=1$ and $\gamma=-0.96$ and the initial status of $u_{6}$.

$v(x, t)=\tilde{v}(\xi)$, where $\xi$ is as defined previously, into (6.1) and balancing the highest order derivative and non-linear terms, we obtain $N_{u}=1$ and $N_{v}=2$. If candidate traveling wave solutions are chosen according to (3.2), then all of the coefficients are required to be zero. Accordingly, we will use the more general form (3.9) and consider candidate solutions

$$
\begin{aligned}
& \tilde{u}(\xi)=\hat{c}_{0,0}+\frac{\hat{\mathrm{a}}_{1,1} F}{\theta F(\xi)+\hat{c}_{2,1} F^{\mathrm{I}}(\xi)} \\
& \tilde{v}(\xi)=\tilde{\boldsymbol{c}}_{0,0}+\frac{\tilde{\mathrm{a}}, 1_{, 1} F(\xi)+\tilde{\boldsymbol{c}}_{2,1} F^{\mathrm{I}}(\xi)}{\theta F(\xi)+1}+\frac{\tilde{\mathrm{a}}, 2_{2} F^{2}(\xi)+\tilde{\boldsymbol{a}}_{2,2} F(\xi) F^{\mathrm{I}}(\xi)}{(\theta F(\xi)+1)^{2}}
\end{aligned}
$$

where $F$ satisfies $\operatorname{ODE}(2.1)$ with coefficients $q_{k}, k=0, \ldots, 4$. By substituting (6.2) into (6.1), we can ascertain the following sufficient conditions for $\tilde{u}$ and $\tilde{v}$ to satisfy the shallow long wave approximate equations (6.1):

$$
\begin{aligned}
\mu & = \pm \frac{\hat{c}_{1,1}}{\alpha} \\
\hat{c}_{0,0} & =-v+\frac{4 q_{0} \hat{c}_{1,1} \theta^{3}+3 q_{1} \hat{c}_{1,1} \theta^{2}-2 q_{2} \hat{c}_{1,1} \theta+q_{3} \hat{c}_{1,1}}{4 \alpha^{2}} \\
\hat{c}_{2,1} & =0
\end{aligned}
$$




$$
\begin{aligned}
& \tilde{c}_{0,0}=\frac{\hat{c}^{2} \mid}{16 \alpha^{4}} 12 q_{0} q_{1} \theta^{5}-8 q_{0}^{2} \theta^{6}-\left(12 q_{0} q_{2}+3 q_{1}^{2}\right) \theta^{4}+\left(16 q_{0} q_{3}+4 q_{1} q_{2}\right) \theta^{3} \\
& \tilde{c}_{1,1}=\frac{\hat{c}_{1,1}^{2}\left(4 \theta^{3} q_{0}-3 \theta^{2} q_{1}+2 \theta q_{2}-q_{3}\right)}{4 \alpha^{2}}, \\
& \tilde{c}_{1,2}=-\frac{\hat{c}^{2}}{\hat{c}^{2}}, \\
& \tilde{c}_{2,1}= \pm \frac{1,1}{2 \alpha}, \\
& \tilde{c}_{2,2}=\mp \frac{\hat{c}^{\ngtr, 1}}{2 \alpha}
\end{aligned}
$$

where $\alpha={ }^{\prime} \backslash \overline{q_{0} \theta^{4}-q_{1} \theta^{3}+q_{2} \theta^{2}-q_{3} \theta+q_{4}}$ and $\theta, v, \hat{c}_{1,1}$ are arbitrary constants. Note that these requirements are the same as those reported in [2]. Note also that there are no conditions restricting the choice of coefficients $q_{k}, k=0, \ldots, 4$, of ODE (2.1). Using $\phi_{j, k}(\cdot, 0), j=1, \ldots, 6, k=1, \ldots, 4$, from Theorem 1 , we can reproduce the same Jacobian elliptic solutions of (6.1) reported in [2]. We also can deduce many new solutions by applying Theorems 1-3. These solutions cannot be obtained using the results in [2]. For example, choosing $\theta=0$ and $q_{j}=p_{7, j}(\gamma), j=0, \ldots, 4$, we can obtain the following solutions for the shallow long wave approximate equations (6.1):

$$
\begin{aligned}
u_{j}(x, t)=-v & +\frac{\vartheta \beta}{\mathrm{J} \alpha^{2}}+\vartheta \phi_{7, j}(\mu(x-v t), j=1 \ldots, 4, \\
v_{j}(x, t)=-\vartheta^{2} & +\frac{\beta}{\eta} \phi_{7, j} \mu(x-v t)-\frac{1}{2 \phi^{1}}(\mu(x-v t) \\
& \left.16 \alpha^{4}\right)^{7, j} \\
& +\frac{-}{2} \phi_{7, j}^{2}\left(\mu(x-v t)^{\prime}, \quad j=1 \ldots, 4,\right.
\end{aligned}
$$

where $\phi_{7, j}, j=1, \ldots, 4$, are as defined in Section 2, $\alpha={ }^{\prime} \overline{\gamma^{3}\left(1-m^{2}\right)+\gamma^{2}\left(2-m^{2}\right)+\gamma}$, $\beta=\gamma^{2}\left(3 m^{2}-3\right)+\gamma\left(2 m^{2}-4\right)-1, \eta=\gamma^{4}\left(3 m^{4}-6 m^{2}+3\right)+\gamma^{3}\left(4 m^{4}-12 m^{2}+8\right)+$ $\gamma^{2}\left(6-6 m^{2}\right)-1, \mu=\vartheta / \alpha$, and $v, \gamma, \vartheta$ and $m(m \in(0,1))$ are arbitrary. For the other solutions, we leave it to the reader.

To show the physical insight of these solutions, we take the solution $\left(u_{1}, v_{1}\right)$ as an example. Figures 5 and 6 display the graphs of $u_{1}$ and $v_{1}$ with $m=0.99, v=-2$ and 

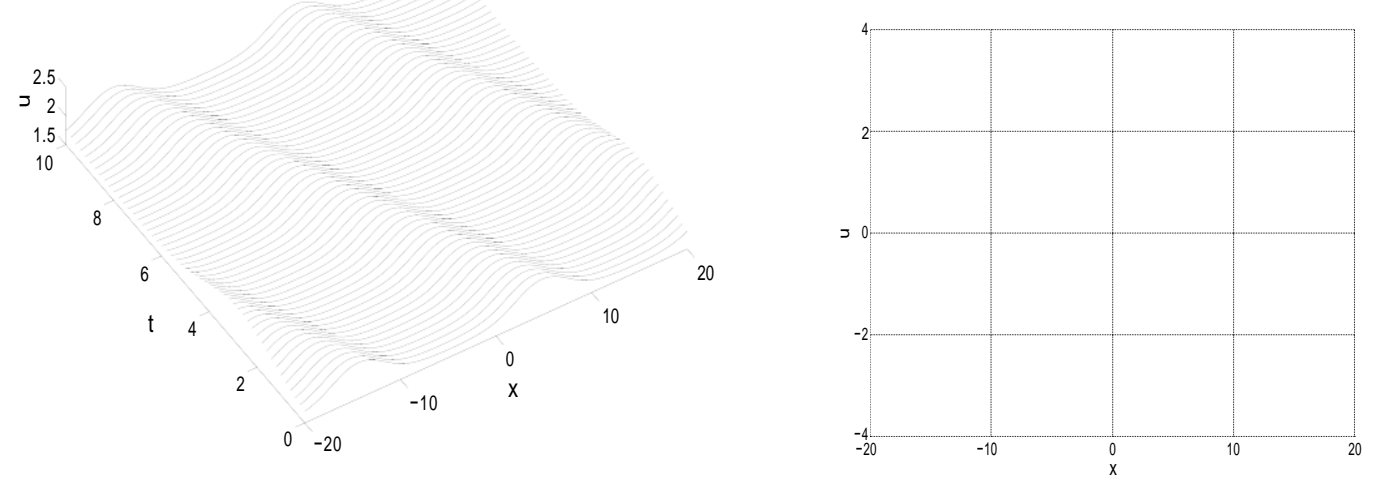

Figure 5: The plot of the solution $u_{1}$ of the shallow long wave approximate equations (6.1) with $m=0.99, v=-2$ and $\vartheta=\gamma=1$ and the initial status of $u_{1}$.

$\vartheta=\gamma=1$. Clearly, the solution describes the propagation of waves with horizontal velocity $u_{1}$ along the negative $x$-direction.

\section{Conclusion}

In this paper, we have presented a generalized expansion method for generating traveling wave solutions of non-linear partial differential equations. This method has been successfully applied to the Boussinesq equation, the modified KdV equation and the shallow long wave approximate equations, and many new results have been obtained. For each equation investigated, we are able to replicate solutions previously derived in the literature, and discover many new ones. Extensions to two and three dimensional partial differential equations are possible. Other non-linear partial differential equations can be tackled if an appropriate transformation can be found. For example, in [6], the transformation $u=\ln v$ was applied to the Dodd-Bullough-Mikhailov equation to yield a non-linear partial differential equation involving powers of $v$ and its derivatives. 

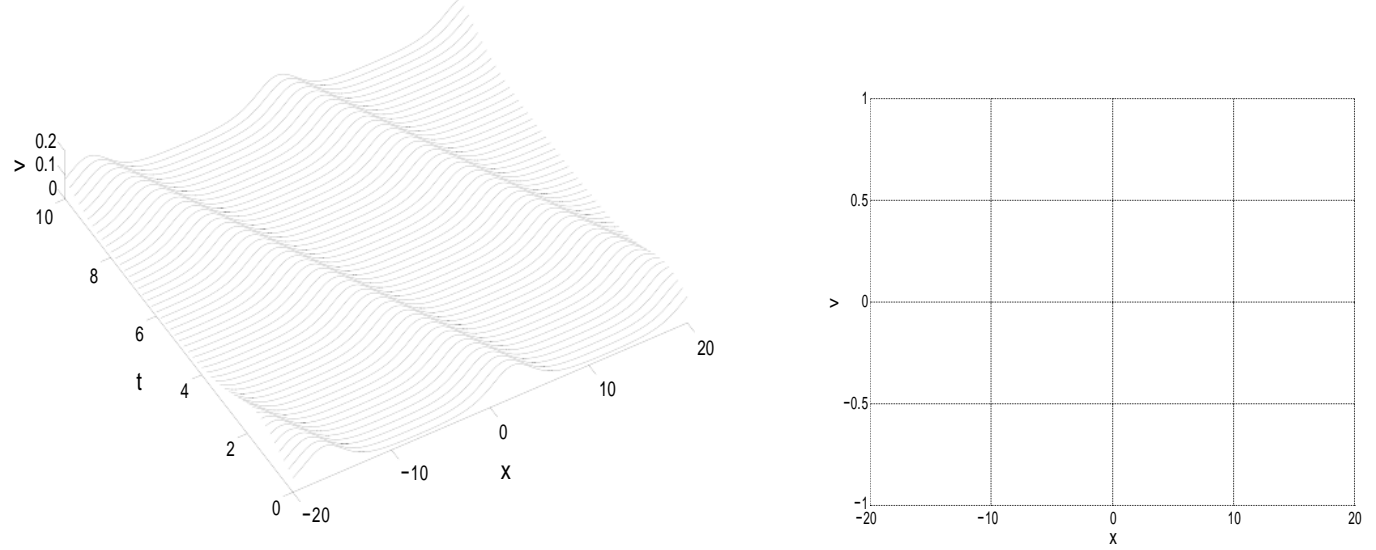

Figure 6: The plot of the solution $v_{1}$ to the shallow long wave approximate equations (6.1) with $m=0.99, v=-2$ and $\vartheta=\gamma=1$ and the initial status of $v_{1}$.

\section{Acknowledgement}

The authors wish to acknowledge the reviewers for their comments and suggestions which have led to significant improvement of the paper.

\section{References}

[1] M. Abramowitz, I. A. Stegun, Handbook of mathematical functions with formulas, graphs, and mathematical tables, Washington DC: National Bureau of Standards, (tenth printing), 1972.

[2] Y. Chen, Q. Wang, A new elliptic equation rational expansion method and its application to the shallow long wave approximate equations, Applied Mathematics and Computaion, 173 (2006) 1163-1182.

[3] M. F. El-Sabbagh, A. T. Ali, New generalized Jacobi elliptic function expansion method, Communications in Nonlinear Science and Numerical Simulation, (2007), doi:10.1016/j.cnsns.2007.04.014. 
[4] E. Fan, Multiple travelling wave solutions of non-linear evolution equations using a unified algebraic method, Journal of Physics A:Mathematical and General, 35 (2002) 6853-6872.

[5] I. S. Gradshteyn, I. M. Ryzhik, Table of Integrals, Series, and Products, San Diego, California: Academic Press, 1980.

[6] J. H. He, X. H. Wu, Exp-function method for nonlinear wave equations, Chaos, Solitons and Fractals, 30 (2006) 700-708.

[7] S. Lai and Y. H. Wu, The asymptotic solution of the Cauchy problem for a generalized Boussinesq equation, Discrete and Continuous Dynamical Systems - Series B, 3 (2003) 401-408.

[8] S. Lai, Y. H. Wu and X. Yang, The global solution of an initial boundary value problem for the damped Boussinesq equation, Communications on Pure and Applied Analysis, 3 (2004) 319-328.

[9] S. Lai, Y. H. Wu and B. Wiwatanapataphee, On exact travelling wave solutions for two types of nonlinear $K(n, n)$ equations and a generalized KP equation, Journal of Computational and Applied Mathematics, 212 (2008) 291-299.

[10] S. Lai, Y. H. Wu and Y. Zhou, Some physical structures for the $(2+1)$-dimensional Boussinesq water equation with positive and negative exponents, Computers and Mathematics with Applications, 56 (2008) 339-345.

[11] Q. Lin, Y. H. Wu, R. Loxton and S. Lai, Linear B-spline finite element method for the improved Boussinesq equation, Journal of Computational and Applied Mathematics (2008), doi:10.1016/j.cam.2008.05.049

[12] S. Liu, Z. Fu, S. Liu, Q. Zhao, Jacobi elliptic function expansion method and periodic wave solutions of nonlinear wave equations, Physics Letters A, 289 (2001) 69-74. 
[13] J. Liu, L. Yang, K. Yang, Nonlinear transformation and Jacobi elliptic function solutions of nonlinear equations, Chaos, Solitons and Fractals, 20 (2004) 11571164.

[14] X. Lv, S. Lai, Y. H. Wu, An auxiliary equation technique and exact solutions for a nonlinear Klein-Gordon equation, Chaos, Solitons and Fractals, (2007), doi:10.1016/j.chaos.2007.11.013.

[15] W. Malfliet, The tanh method: I. exact solutions of nonlinear evolution and wave equations, Physica Scripta, 54 (1996) 563-568.

[16] W. Malfliet, The tanh method : a tool for solving certain classes of nonlinear evolution and wave equations, Journal of Computional and Applied Mathematics, 164-165(2004) 529-541.

[17] J. Nickel, Elliptic solutions to a generalized BBM equation, Physics Letters A, 364 (2007), 221-226.

[18] S. Shen, Z. Pan, A note on the Jacobi elliptic function expansion method, Physics Letters A, 308 (2003) 143-148.

[19] A. Wazwaz, New hyperbolic schemes for reliable treatment of Boussinesq equation, Physics Letters A, 358 (2006) 409-413.

[20] X. H. Wu, J. H. He, Solitary solutions, periodic solutions and compacton-like solutions using the Exp-function method, Computers and Mathematics with Applications, 54 (2007) 966-986.

[21] Z. Yan, Jacobi elliptic function solutions of nonlinear wave equations via the new sinh-Gordon equation expansion method, Journal of Physics A, 36 (2003), 19611972. 
[22] H. Zhang, Extended Jacobi elliptic function expansion method and its applications, Communications in Nonlinear Science and Numerical Simulation, 12 (2007) 627-635.

[23] H. Zhang, New exact Jacobi elliptic function solutions for some nonlinear evolution equations, Chaos, Solitons and Fractals, 32 (2007) 653-660.

\section{Appendix}

Some solutions of the Boussinesq equation (4.1), derived from this work, have been given in Section 4, and the rest are listed below.

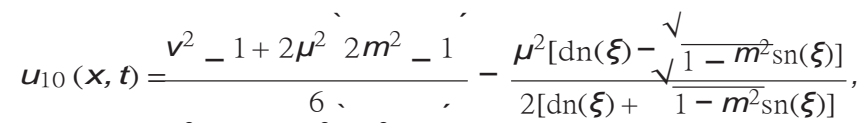

$$
\begin{aligned}
& u_{11}(x, t)=\frac{v^{2}-1-2 \mu^{2} m^{2}+1}{6}+\frac{\mu^{2}\left(1-m^{2}\right)\left[1-m_{\mathrm{sn}}(\xi)\right]}{2\left[1+m_{\mathrm{sn}}(\xi)\right]}, \\
& u_{12}(x, t)=\frac{v^{2}-1-2 \mu^{2} m^{2}+1}{6},-\frac{\mu^{2}\left(1-m^{2}\right)[1-\operatorname{sn}(\xi)]}{2[1+\operatorname{sn}(\xi)]}, \\
& u_{13}(x, t)=\frac{v^{2}-1-2 \mu^{2} m^{2}+1}{6}-\frac{\mu^{2}\left(1-m^{2}\right)[\operatorname{dn}(\xi)-\operatorname{cn}(\xi)]}{2[\operatorname{dn}(\xi)+\operatorname{cn}(\xi)]}, \\
& u_{14}(x, t)=\frac{v^{2}-1-2 \mu^{2} m^{2}+1}{6},+\frac{\mu^{2}\left(m^{2}-1\right)\left[m_{\mathrm{cn}}(\xi)-\mathrm{dn}(\xi)\right]}{2\left[m_{\mathrm{cn}}(\xi)+\operatorname{dn}(\xi)\right]}, \\
& u_{15}(x, t)=\frac{v^{2}-1-2 \mu^{2} m^{2}-2}{6}-\frac{\mu^{2} m^{2}\left[\operatorname{dn}(\xi)-\bar{v} 1-m^{2}\right]}{2\left[\operatorname{dn}(\xi)+\overline{\left.1-m^{2}\right]}\right.}, \\
& u_{16}(x, t)=\frac{v^{2}-1-2 \mu^{2} m^{2}-2}{6 \cdot}-\frac{\mu^{2} m^{2}[1-\operatorname{dn}(\xi)]}{2[1+\operatorname{dn}(\xi)]}, \\
& u_{17}(x, t)=\frac{v^{2}-1-\mu^{2} 2 m^{2}+12 m+2}{6}
\end{aligned}
$$

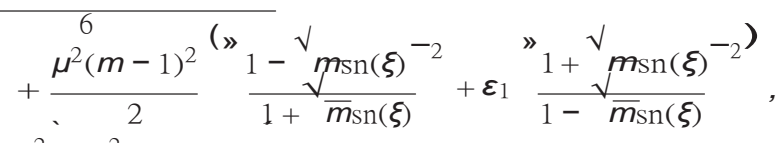

$$
\begin{aligned}
& u_{18}(x, t)=\frac{v^{2}-1-\mu^{2} 2 m^{2}+12 m+2}{\mu^{2}(m-1)^{2}(\gg \operatorname{dn}(\zeta)}
\end{aligned}
$$

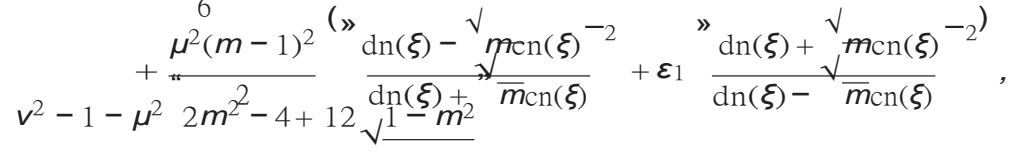

$$
\begin{aligned}
& u_{19}(x, t)=
\end{aligned}
$$

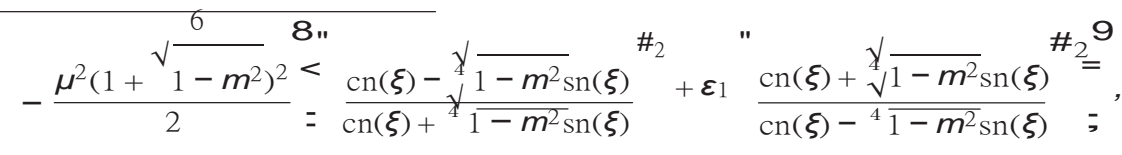




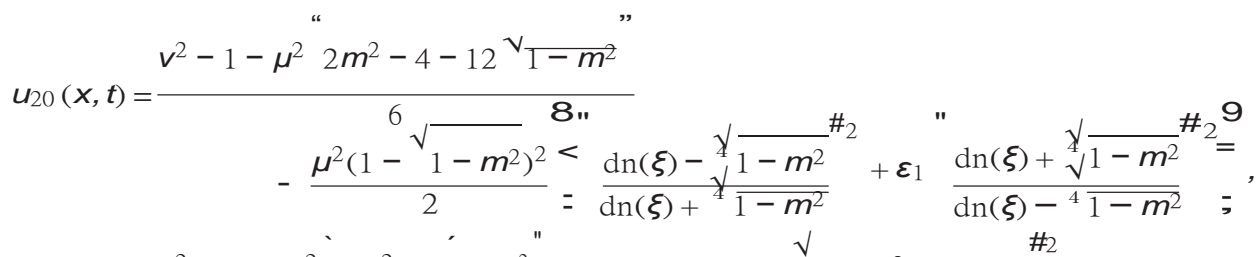

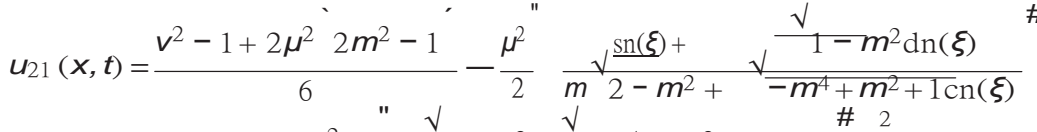

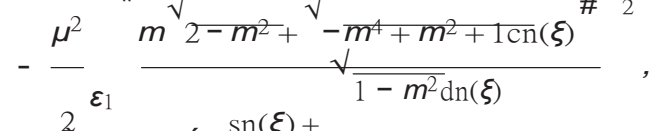

$$
\begin{aligned}
& u_{22}(x, t)=\frac{v^{2}-1+2 \mu^{2} 2 m^{2}-1}{6}
\end{aligned}
$$

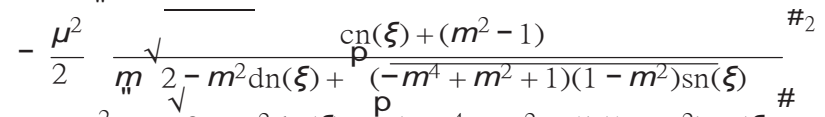

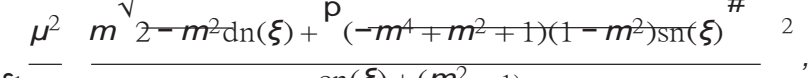

$$
\begin{aligned}
& -\varepsilon_{1}-\frac{\operatorname{cn}(\xi)+\left(m^{2}-1\right) \quad \text { \#2 }}{"} \text {, }
\end{aligned}
$$

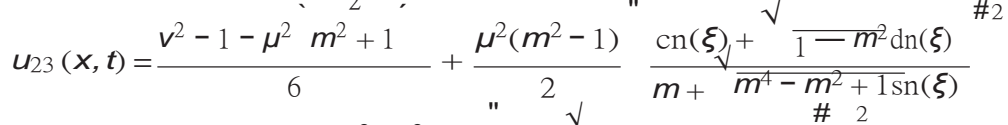

$$
\begin{aligned}
& +\varepsilon_{1} \frac{\mu^{2}\left(m^{2}-1\right)}{2} \frac{m+\sqrt{m^{4}-m^{2}+1 \mathrm{sn}(\xi)}{ }^{\#}}{\mathrm{cn}(\xi)+{ }_{n} \overline{1-m^{2}} \mathrm{dn}(\xi)} \\
& u_{24}(x, t)=\frac{v^{2}-1-\mu^{2} m^{2}+1}{6}-\frac{\mu^{2}\left(1-m^{2}\right)^{2}}{\sqrt{ }^{2}} \frac{\operatorname{sn}(\xi)+\sqrt{1-m^{2}}}{m \operatorname{dn}(\xi)+{ }_{m^{4}-m^{2}+1 \mathrm{cn}(\xi)}} \#_{2} \\
& \mu^{2} m \operatorname{dn}(\xi)+m^{4}-m^{2}+1 \mathrm{cn}(\xi){ }^{2}, \\
& -\varepsilon_{1}-\overline{\operatorname{sn}(\xi)+\overline{\overline{1-m^{2}}}}
\end{aligned}
$$

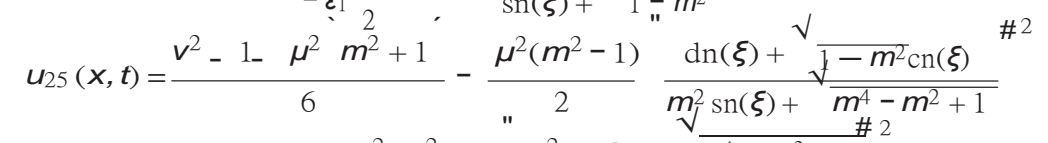

$$
\begin{aligned}
& -\varepsilon_{1} \frac{\mu^{2}\left(m^{2}-1\right)}{2} \frac{m^{2} \operatorname{sn}(\xi)+{ }^{2}}{\ln (\xi)+{ }^{4}-m^{2}+1} \\
& u_{26}(x, t)=\frac{v^{2}-1-\mu^{2} m^{2}+1^{\prime 2}}{6}+\frac{\mu^{2}\left(1-m^{2}\right)^{2}}{2} \frac{1+m \sqrt{ } \sqrt{1-m^{2} \operatorname{sn}(\xi)}}{\sqrt{m^{4}-m^{2}+1 \mathrm{~d} n}(\xi)} \\
& +\varepsilon_{1} \frac{\mu^{2}}{2} \frac{m^{2} \operatorname{cn}(\xi)+{\sqrt{m^{4}-m^{2}}+1 \mathrm{dn}(\xi)}^{\# 2}}{1+m 1-m^{2} \operatorname{sn}(\xi)}
\end{aligned}
$$

where, for each $j=1,2, \varepsilon_{j} \in\{0,1\}$, and $\mu$ and $v$ are arbitrary real constants. Note that the solutions $u_{j}, j \in\{11,14,15,16,17,18,25,26\}$, are bounded, while the solutions $u_{j}, j \in\{12,13,19,20,21,22,23,24\}$, are unbounded. 